\title{
Macrocycle Ring Expansion by Double Stevens Rearrangement
}

\author{
K. K. Ellis-Holder, B. P. Peppers, A. Y. Kovalevsky and \\ Steven T. Diver* \\ Department of Chemistry, University at Buffalo-SUNY, Amherst, NY, \\ 14260-3000
}

\section{SUPPORTING INFORMATION}

Table of Contents

A. General Experimental S2

B. Experimental Procedures S3

C. NMR Spectra

1. ${ }^{1} \mathrm{H}$ and ${ }^{13} \mathrm{C}$ NMR Data for Compounds 2-3 S8

2. ${ }^{1} \mathrm{H}$ and ${ }^{13} \mathrm{C}$ NMR Data for Compounds 7-9A $\quad \mathrm{S} 16$ 


\section{General Experimental}

Reactions were conducted under argon atmosphere unless otherwise noted. Benzene was distilled from $\mathrm{CaH}_{2}$ immediately prior to use. Xylene (mixture of isomers) was distilled from $\mathrm{CaH}_{2}$ and stored over $4 \AA$ 年 molecular sieves. GC mass spectra were obtained on a benchtop HP gc/mass spectrometer. ${ }^{1} \mathrm{H}$ NMR and ${ }^{13} \mathrm{C}$ NMR were recorded in $\mathrm{CDCl}_{3}$ or acetone- $d_{6}$ at the indicated frequency. ${ }^{1} \mathrm{H}$ NMR spectra were referenced at 0 ppm on the TMS signal and ${ }^{13} \mathrm{C}$ NMR spectra were referenced at $77.0 \mathrm{ppm}$ for $\mathrm{CDCl}_{3}$. Spectra in acetone- $d_{6}$ were referenced at the residual acetone signal which was at 2.04 ppm and $206.0 \mathrm{ppm}$ for ${ }^{1} \mathrm{H}$ and ${ }^{13} \mathrm{C}$ NMR, respectively. Ethyl diazomalonate, ${ }^{1}$ 1,3bis(methoxy)-2-imidazolidinone, ${ }^{2}$ 1,3-bis(methoxy)-2-benzimidazolidinone ${ }^{3}$ and dithiols were synthesized by literature methods.

\footnotetext{
${ }^{1}$ Regitz, M.; Liedhegener, A., Chem. Berichte 1966, 99, 3128-4.

${ }^{2}$ Petersen, H.; Reuther, W. Liebigs Ann. 1972, 766, 58.

${ }^{3}$ Zinner, H.; Spangenberg, B. Liebigs Ann. 1958, 91, 1432.
} 


\section{4, 11-Dithiabenzimidazolophane $2 \mathrm{~A}$}

A 1 L 2-necked rb flask equipped with a reflux condensor, magnetic stir bar and an addition funnel was charged with $12 \mathrm{~g}(36 \mathrm{mmol})$ of 3,10-dithia-benzimidazolophane $\mathbf{1 A},{ }^{4} 790 \mathrm{mg}(1.8 \mathrm{mmol})$ of $\mathrm{Rh}_{2}(\mathrm{OAc})_{4}$ and $350 \mathrm{~mL}$ xylenes and brought to reflux under an argon atmosphere. The addition funnel was loaded with $14 \mathrm{~g}(76 \mathrm{mmol})$ of diethyldiazomalonate in $50 \mathrm{~mL}$ xylenes and this was added over a period of $1.5 \mathrm{~h}$ to the $\mathrm{h}$. After addition was complete, refluxing was continued for another $2 \mathrm{~h}$ at which time TLC indicated full consumption of starting materials. Solvents were removed in vacuo and the crude mass was loaded onto a silica gel column. The product was eluted with $25 \% / 25 \% / 50 \% \mathrm{v} / \mathrm{v} / \mathrm{v}$ ethyl acetate-chloroform-hexanes to give $12 \mathrm{~g} \mathbf{2 A}(51 \%)$ as a white solid. Crystals of $\mathbf{2 A}$ were obtained by slow evaporation from $\mathrm{CH}_{2} \mathrm{Cl}_{2}$ and diethyl ether, mp $160^{\circ} \mathrm{C} .{ }^{1} \mathrm{H}$ NMR $\left(500 \mathrm{MHz}, \mathrm{CDCl}_{3}, \mathrm{ppm}\right) 7.22-7.18(\mathrm{~m}, 6 \mathrm{H}), 6.06$ (br s, 2H), 4.85 (br d, $J=76 \mathrm{~Hz}, 4 \mathrm{H}), 4.44-4.30(\mathrm{~m}, 8 \mathrm{H}), 3.72(\mathrm{~d}, J=15.5 \mathrm{~Hz}, 2 \mathrm{H}), 3.01$ (br d, $J=15$ $\mathrm{Hz}, 2 \mathrm{H}), 1.39-1.34(\mathrm{~m}, 12 \mathrm{H}) .{ }^{13} \mathrm{C} \mathrm{NMR}\left(125.7 \mathrm{MHz}, \mathrm{CDCl}_{3}, \mathrm{ppm}\right) 169.4,167.5,151.6$, 132.63, 129.2 (broad signal), $128.9121 .6(\mathrm{CH}), 108.2(\mathrm{CH}), 63.0(\mathrm{CH} 2), 62.9(\mathrm{CH} 2)$, 42.7, 39.2 (broad signal), 14.0 (CH3), 13.9 (CH3). FT-IR (thin film, $\mathrm{cm}^{-1}$ ) 2982, 2936, $1725,1619,1517,1494,1424,1388,1367,1245,1201,1157,1095,1027,860$, 816. High resolution MS (EI, $m / z$ ): anal calcd for $\mathrm{C}_{31} \mathrm{H}_{36} \mathrm{~N}_{2} \mathrm{~S}_{2} \mathrm{O}_{9}$ : 644.1862; found: 644.1817, error: 7 ppm. Elemental analysis, calculated: $\mathrm{C}, 57.8 ; \mathrm{H}, 5.6 ; \mathrm{N}, 4.3 \%$; found: $\mathrm{C}, 57.7 ; \mathrm{H}, 5.6$; N, $4.3 \%$.

Crystals of $\mathbf{2 A}$ were subjected to x-ray analysis. See attached cif file.

\section{4, 11-Dithiabenzimidazolophane 2B}

A $50 \mathrm{~mL}$ Schlenk flask equipped with a coldfinger condenser, magnetic stir bar and a rubber septum was charged with $70 \mathrm{mg}(0.2 \mathrm{mmol})$ dithiabenzimidazolophane $\mathbf{1 B},{ }^{4}$ $2.2 \mathrm{mg}(0.005 \mathrm{mmol})$ of $\mathrm{Rh}_{2}(\mathrm{OAc})_{4}$ and $2 \mathrm{~mL}$ xylenes and brought to reflux under an argon atmosphere. Diethyl diazomalonate $(112 \mathrm{mg}, 0.6 \mathrm{mmol})$ was dissolved in $2 \mathrm{~mL}$ xylenes and this solution was added over a period of $1.5 \mathrm{~h}$ to the reaction mixture. After addition was complete, reflux was continued for another $2 \mathrm{~h}$ at which point TLC indicated full consumption of starting materials. Solvents were removed in vacuo and the crude mass was loaded onto a silica gel column $(1 \mathrm{~cm} \mathrm{x} 4 \mathrm{~cm})$. The product was eluted with 1:3 ethyl acetate:hexanes to give $78 \mathrm{mg}$ of a colorless oil which yielded $56 \mathrm{mg} \mathbf{2 B}$ $(42 \%)$ as a white solid upon trituration with diethyl ether from a dichloromethane solution, mp $179-180^{\circ} \mathrm{C}$. Analytical TLC $\left(20 \%\right.$ ethyl acetate/hexanes) $\mathrm{R}_{f} 0.25 .{ }^{1} \mathrm{H}-\mathrm{NMR}$ $\left(500 \mathrm{MHz}, \mathrm{CDCl}_{3}\right.$, ppm) $\delta 7.18-7.15(\mathrm{~m}, 2 \mathrm{H}), 7.10-7.06(\mathrm{~m}, 2 \mathrm{H}), 6.94-6.94$ (br m, 2H), 6.74 (br s, 1H), 5.00 (br d, $J=15.5 \mathrm{~Hz}, 2 \mathrm{H}$ ), 4.82 (br s, $1 \mathrm{H}), 4.69$ (d, $J=15.5 \mathrm{~Hz}$, $1 \mathrm{H}), 4.46-4.27(\mathrm{~m}, 8 \mathrm{H}), 3.59$ (br d, $J=14 \mathrm{~Hz}, 1 \mathrm{H}), 3.49-3.45(\mathrm{~m}, 2 \mathrm{H}), 3.11$ (br d, $J=$ $17 \mathrm{~Hz}, 1 \mathrm{H}), 2.35(\mathrm{~s}, 3 \mathrm{H}), 1.38-1.33(\mathrm{~m}, 12 \mathrm{H}), 1.26-1.19(\mathrm{~m}, 3 \mathrm{H}) .{ }^{13} \mathrm{C}$ NMR $(125.7$ $\mathrm{MHz}, \mathrm{CDCl}_{3}$, ppm) 169.4, 169.1, 168.3, 168.2, 135.1, 133.5, 131.9, 130.8, 130.0, 128.9, $128.5,121.4(3), 121.4(0), 108.4,108.1,62.8,62.7,41.8,35.7,34.9,31.5,22.6,20.2$, 17.6, 14.1, 14.0, 13.9(2), 13.9(1), 13.8. FT-IR (thin film, $\mathrm{cm}^{-1}$ ) 2977, 2934, 1728, 1618,

\footnotetext{
${ }^{4}$ Ellis, K. K.; Wilke, B.; Zhang, Y.; Diver, S. T. Org. Lett. 2000, 2, 3785-3788.
} 
1489, 1426, 1387, 1242, 1156, 1097, 1027, 858, 753. High resolution MS (EI, $m / z)$ : anal calcd for $\mathrm{C}_{33} \mathrm{H}_{40} \mathrm{~N}_{2} \mathrm{~S}_{2} \mathrm{O}_{9}: 672.2177$; found: $673.2248\left(\mathrm{M}+\mathrm{H}^{+}\right)$, error: $1.6 \mathrm{ppm}$.

\section{[3.3] Benzimidazolophane 3A}

Dithiacyclophane 2A (4.5 g, $7 \mathrm{mmol}$ ) was dissolved in $200 \mathrm{~mL}(\mathrm{EtO})_{3} \mathrm{P}$ by heating to $60{ }^{\circ} \mathrm{C}$ for about $10 \mathrm{~min}$ and was then transferred to an immersion well reactor, equipped with a medium pressure $\mathrm{Hg}$ lamp (Hanovia) in a pyrex water-jacketed cold finger. The solution was sparged with argon during UV irradiation. Irradiation was conducted for $2 \mathrm{~h}$ at which time, TLC showed full conversion of the starting material. The solvent was removed in vacuo in a fume hood and the residue was rinsed with ice cold diethyl ether yielding $1.89 \mathrm{~g}(46 \%)$ of $\mathbf{3 A}$ as a white solid. This was rinsed several times with more diethyl ether and observed to be pure by NMR. Crystals were grown by slow evaporation from $\mathrm{CH}_{2} \mathrm{Cl}_{2}$ and diethyl ether, mp 141-143 ${ }^{\circ} \mathrm{C} .{ }^{1} \mathrm{H}$ NMR $(500 \mathrm{MHz}$, $\left.\mathrm{CDCl}_{3}, \mathrm{ppm}\right) \delta 7.23(\mathrm{~s}, 2 \mathrm{H}), 7.01-6.93(\mathrm{~m}, 4 \mathrm{H}), 6.44(\mathrm{~s}, 2 \mathrm{H}), 4.82(\mathrm{~d}, J=15 \mathrm{~Hz}, 2 \mathrm{H})$, 4.44-4.31 (m, 8H), $4.13(\mathrm{~d}, J=15 \mathrm{~Hz}, 2 \mathrm{H}), 3.48(\mathrm{~d}, J=15 \mathrm{~Hz}, 2 \mathrm{H}), 3.36(\mathrm{~d}, J=15 \mathrm{~Hz}$, 2H), 1.39-1.32 (m, 12H). ${ }^{13} \mathrm{C}$ NMR (125.7 MHz, $\left.\mathrm{CDCl}_{3}, \mathrm{ppm}\right) \delta 171.9,170.3,152.5$, $133.4(\mathrm{CH}), 130.1(\mathrm{CH}), 129.5,127.6,120.9(\mathrm{CH}), 108.2(\mathrm{CH}), 62.4\left(\mathrm{CH}_{2}\right), 62.2\left(\mathrm{CH}_{2}\right)$, 57.5, $43.1\left(\mathrm{CH}_{2}\right), 38.4\left(\mathrm{CH}_{2}\right), 13.9\left(\mathrm{CH}_{3}\right), 13.8\left(\mathrm{CH}_{3}\right)$. FT-IR (thin film, $\left.\mathrm{cm}^{-1}\right)$ 2978, 2359 , $1731,1495,1440,1365,1300,1236,1114,1095,1081,860$, 772. High resolution MS (EI, $m / z)$ : anal calcd for $\mathrm{C}_{31} \mathrm{H}_{36} \mathrm{~N}_{2} \mathrm{O}_{9}$ : 580.2445; found: 580.2420; error: $4.3 \mathrm{ppm}$. Elemental analysis, calculated: C, 64.1; H, 6.3; N, 4.8\%; found, C, 63.9; H, 6.3; N, 4.8\%.

Crystals of $\mathbf{3 A}$ were subjected to x-ray analysis. See attached cif file.

\section{[3.3] Benzimidazolophane 3B}

Dithiacyclophane 2B (50 mg, $0.07 \mathrm{mmol})$ was dissolved in $5 \mathrm{~mL}(\mathrm{EtO})_{3} \mathrm{P}$ by heating to $60{ }^{\circ} \mathrm{C}$ for about $10 \mathrm{~min}$ and was then transferred to a quartz photolysis tube with a constant sparge of argon. A medium pressure $\mathrm{Hg}$ lamp (Hanovia) in a pyrex water-jacketed cold finger was employed for side-on photolysis. Irradiation was conducted for $3 \mathrm{~h}$, at which time full conversion of starting material was observed (TLC). The solvent was removed in vacuo in a fume hood and the residue was rinsed with cold diethyl ether yielding $14 \mathrm{mg}(31 \%)$ of $\mathbf{3 B}$ as a white solid, mp $130-131{ }^{\circ} \mathrm{C} .{ }^{1} \mathrm{H}$ NMR $\left(500 \mathrm{MHz}, \mathrm{CDCl}_{3}, \mathrm{ppm}\right) 7.08-7.06(\mathrm{~m}, 1 \mathrm{H}), 6.98-6.94(\mathrm{~m}, 2 \mathrm{H}), 6.89(\mathrm{~s}, 1 \mathrm{H}), 6.86-$ $6.84(\mathrm{~m}, 1 \mathrm{H}), 6.75(\mathrm{~s}, 1 \mathrm{H}), 5.12(\mathrm{~d}, J=15.5 \mathrm{~Hz}, 1 \mathrm{H}), 4.82(\mathrm{~d}, J=15.5 \mathrm{~Hz}, 1 \mathrm{H}), 4.60-$ $4.53(\mathrm{~m}, 1 \mathrm{H}), 4.54-4.23(\mathrm{~m}, 8 \mathrm{H}), 4.14-4.06(\mathrm{~m}, 2 \mathrm{H}), 3.85(\mathrm{~d}, J=15.5,1 \mathrm{H}), 3.61(\mathrm{~d}, J$ $=16.5,1 \mathrm{H}), 3.22-3.12(\mathrm{~m}, 2 \mathrm{H}), 2.35(\mathrm{~s}, 3 \mathrm{H}), 1.44-1.31(\mathrm{~m}, 12 \mathrm{H}), 1.27(\mathrm{~s}, 3 \mathrm{H}) .{ }^{13} \mathrm{C}$ NMR (125.7 MHz, $\left.\mathrm{CDCl}_{3}, \mathrm{ppm}\right) 172.5,171.9,171.2,170.4,153.4,135.5,132.9,132.4$, $131.1,130.2$, 129.7, 129.4, 120.9, 120.8, 108.6, 108.2, 62.6, 62.4(3), 62.4(0), 62.2, 58.0, 57.8, 43.9, 42.2, 32.9, 32.5, 20.3, 17.7, 14.0(4), 14.0(1), 13.9. FT-IR (thin film, $\mathrm{cm}^{-1}$ ) 2979, 2363, 2341, 1729, 1493, 1446, 1366, 1236, 1091, 1029, 859. High resolution MS (EI, $m / z$ ): anal calcd for $\mathrm{C}_{33} \mathrm{H}_{40} \mathrm{~N}_{2} \mathrm{O}_{9}$ : 608.2735; found: $631.2623\left(\mathrm{M}+\mathrm{Na}^{+}\right)$; error: 0.5 ppm. 


\section{3,9-Dithiaimidazolidinophane 7A}

A $500 \mathrm{~mL}$ rb flask equipped with a magnetic stirbar and rubber septum was charged with $150 \mathrm{~mL}$ methanol and 15 drops of $\mathrm{H}_{2} \mathrm{SO}_{4}$ and stirred at room temperature under an argon atmosphere. Two gas tight syringes $(5 \mathrm{~mL})$ with teflon-tipped plungers were individually loaded with solutions of $348 \mathrm{mg}$ of 1,3-bis(methoxy)-2imidazolidinone $(2 \mathrm{mmol})$ and $340 \mathrm{mg}$ of 1,3-benzenedimethanethiol $(2 \mathrm{mmol})$ each in methanol ( $5 \mathrm{~mL}$ total volume), which were added over a period of $6 \mathrm{~h}$ via syringe pump. After the addition was complete, TLC indicated complete consumption of reactants. The contents were diluted with $100 \mathrm{~mL}$ of $\mathrm{CHCl}_{3}$ and transferred into a $500 \mathrm{~mL}$ separatory funnel and washed with $\mathrm{NaHCO}_{3}\left(2 \times 100 \mathrm{~mL}\right.$ portions), dried $\left(\mathrm{K}_{2} \mathrm{CO}_{3}\right)$ and evaporated (rotary evaporator) to give a crude white solid. This was dissolved in a minimum of $\mathrm{CHCl}_{3}$, evaporated onto $2 \mathrm{~g}$ flash-grade silica gel in vacuo (rotary evaporator), then loaded onto a silica gel column $(4 \mathrm{~cm} \times 15 \mathrm{~cm})$ and eluted with $20 \%$ ethyl acetatehexanes to give $377 \mathrm{mg}$ 7A (67 \%) as a white solid. Crystals of 7A were obtained by slow evaporation from $\mathrm{CH}_{2} \mathrm{Cl}_{2}, \mathrm{mp} 159-161{ }^{\circ} \mathrm{C} .{ }^{1} \mathrm{H}$ NMR $\left(500 \mathrm{MHz}, \mathrm{CDCl}_{3}, \mathrm{ppm}\right) \delta 7.34$ $-7.30(\mathrm{~m}, 1 \mathrm{H}), 7.23-7.22(\mathrm{~m}, 2 \mathrm{H}), 7.15(\mathrm{~s}, 1 \mathrm{H}), 5.44(\mathrm{~d}, J=15,2 \mathrm{H}), 3.77(\mathrm{~d}, J=15$ $\mathrm{Hz}, 2 \mathrm{H}), 3.75-3.66(\mathrm{~m}, 4 \mathrm{H}), 2.80-2.78(\mathrm{~m}, 2 \mathrm{H}), 2.67-2.64(\mathrm{~m}, 2 \mathrm{H}) .{ }^{13} \mathrm{C}$ NMR $(125.7$ $\left.\mathrm{MHz}, \mathrm{CDCl}_{3}, \mathrm{ppm}\right) \delta$ 157.5, 139.6, 129.1(CH), $128.0(\mathrm{CH}), 126.9(\mathrm{CH}), 47.9\left(\mathrm{CH}_{2}\right), 38.4$ $\left(\mathrm{CH}_{2}\right), 37.1\left(\mathrm{CH}_{2}\right)$. COSY analysis showed coupling between protons with $\delta 7.23-7.22$ and 3.75 - 3.66; 5.44 and 3.75 - 3.66. FT-IR (thin film, $\mathrm{cm}^{-1}$ ) 1690, 1486, 1439, 1249, $1224,715$. High resolution MS (EI, $m / z)$ : anal calcd for $\mathrm{C}_{13} \mathrm{H}_{16} \mathrm{~N}_{2} \mathrm{~S}_{2} \mathrm{O}$ : 280.0701 ; found: 280.0699, error: $1.0 \mathrm{ppm}$.

\section{3,9-Dithiaimidazolidinophane 7B}

A $500 \mathrm{~mL}$ rb flask equipped with a magnetic stir bar and rubber septum was charged with $150 \mathrm{~mL}$ methanol and 15 drops of $\mathrm{H}_{2} \mathrm{SO}_{4}$ and stirred at room temperature under an argon atmosphere. A gas tight syringe $(10 \mathrm{~mL})$ with teflon-tipped plunger was loaded with a solution of $348 \mathrm{mg}$ of 1,3-bis(methoxy)-2-imidazolidinone $\mathbf{6}(2 \mathrm{mmol})$ and $396 \mathrm{mg}$ of 1,3 benzenedimethanethiol 5B $(2 \mathrm{mmol})$ in methylene chloride $(10 \mathrm{~mL}$ total volume). This solution was added over a period of $6 \mathrm{~h}$ via syringe pump. After the addition was complete, TLC indicated complete consumption of reactants. The contents were diluted with $100 \mathrm{~mL}$ of $\mathrm{CHCl}_{3}$ and transferred to a $500 \mathrm{~mL}$ separatory funnel and washed with $\mathrm{NaHCO}_{3}\left(2 \times 100 \mathrm{~mL}\right.$ portions), dried $\left(\mathrm{K}_{2} \mathrm{CO}_{3}\right)$, and concentrated (rotary evaporator) to give a crude white solid. This was dissolved in a minimum of $\mathrm{CHCl}_{3}$, evaporated onto $2 \mathrm{~g}$ flash-grade silica gel in vacuo (rotary evaporator), then loaded onto a silica gel column $(4 \mathrm{~cm} \times 15 \mathrm{~cm})$ and eluted with $20 \%$ ethyl acetate-hexanes to give 290 $\mathrm{mg}$ 7B (47\%) as a white solid. Crystals of 7B were obtained by slow evaporation from $\mathrm{CH}_{2} \mathrm{Cl}_{2}$, mp $234-236{ }^{\circ} \mathrm{C} .{ }^{1} \mathrm{H}$ NMR $\left(500 \mathrm{MHz}, \mathrm{CDCl}_{3}, \mathrm{ppm}\right) \delta 7.26(\mathrm{~s}, 1 \mathrm{H}), 7.03(\mathrm{~s}, 1 \mathrm{H})$, $6.94(\mathrm{~s}, 1 \mathrm{H}), 5.51(\mathrm{~d}, J=12 \mathrm{~Hz}, 2 \mathrm{H}), 3.82-3.75(\mathrm{~m}, 4 \mathrm{H}), 3.66(\mathrm{~d}, J=14.5 \mathrm{~Hz}, 2 \mathrm{H}), 2.82$ - 2.69 (m, 4H), 2.31 (s, 6H). $\left.{ }^{13} \mathrm{C} \mathrm{NMR} \mathrm{(125.7} \mathrm{MHz,} \mathrm{CDCl}_{3}, \mathrm{ppm}\right) \delta 157.8,134.9,134.3$, $132.5(\mathrm{CH}), 129.2(\mathrm{CH}), 48.0\left(\mathrm{CH}_{2}\right), 38.4\left(\mathrm{CH}_{2}\right), 35.8\left(\mathrm{CH}_{2}\right), 18.6\left(\mathrm{CH}_{3}\right)$. COSY analysis indicates coupling between signals at $\delta 7.03$ and $3.82-3.75 ; 7.03$ and $2.31 ; 6.94$ and 3.66; 6.94 and 2.31; 5.51 and $3.82-3.75 ; 5.51$ and 3.66; $3.82-3.75$ and 2.31. FT-IR 
(thin film, $\mathrm{cm}^{-1}$ ) 1686, 1488, 1435, 1247, 1227, 906. High resolution MS (EI, m/z): anal calcd for $\mathrm{C}_{15} \mathrm{H}_{20} \mathrm{~N}_{2} \mathrm{~S}_{2} \mathrm{O}$ : 308.1007; found: 308.1012, error: 1.4 ppm.

\section{3,9-Dithia-benzimidazolophane 8A}

Into an argon-flushed $50 \mathrm{~mL}$ rb flask equipped with magnetic stirbar and rubber septum was placed $44 \mathrm{mg}$ 1,3-bis(methoxymethyl)-2-benzimidazolidinone $(0.2 \mathrm{mmol})$, $34 \mathrm{mg}$ 1,4-benzenedimethanethiol $(0.2 \mathrm{mmol})$ in $10 \mathrm{~mL} \mathrm{CH}_{2} \mathrm{Cl}_{2}$ at room temperature to which $0.1 \mathrm{~mL} \mathrm{BF}_{3}-\mathrm{OEt}_{2}(0.113 \mathrm{~g}, 0.8 \mathrm{mmol})$ was added dropwise via syringe. The reactants were stirred for $4 \mathrm{~h}$ at room temperature at which time the reaction was complete (TLC). The reaction contents were poured into a separatory funnel containing $50 \mathrm{~mL} \mathrm{NaHCO}$, partitioned, and the aqueous layer was extracted three times with $20 \mathrm{~mL}$ portions of $\mathrm{CH}_{2} \mathrm{Cl}_{2}$. The pooled organics were dried $\left(\mathrm{K}_{2} \mathrm{CO}_{3}\right)$ and concentrated to give 84 $\mathrm{mg}$ of a white foam which was purified by flash chromatography $(1 \mathrm{~cm} \mathrm{x} 12 \mathrm{~cm})$ 1:4 ethyl acetates-hexanes $(100 \mathrm{~mL})$ then gradient elution to $1: 1$ ethyl acetate-hexanes) to afford $53 \mathrm{mg} \mathbf{8 A}$ as a white solid (83\%). Crystallization from $\mathrm{CH}_{2} \mathrm{Cl}_{2}$ gave white crystals, mp $173-174{ }^{\circ} \mathrm{C}$. Analytical TLC: (1:3 ethyl acetate-hexanes) $\mathrm{R}_{f} 0.45$. ${ }^{1} \mathrm{H}$ NMR (300 MHz, $\mathrm{CDCl}_{3}$, ppm) $\delta 7.08(\mathrm{~s}, 1 \mathrm{H}), 6.86(\mathrm{~s}, 4 \mathrm{H}), 6.72(\mathrm{br} \mathrm{d}, J=7.8 \mathrm{~Hz}, 2 \mathrm{H}), 6.60-$ $6.54(\mathrm{~m}, 1 \mathrm{H}), 5.75(\mathrm{~d}, J=14.4 \mathrm{~Hz}, 2 \mathrm{H}), 4.40(\mathrm{~d}, J=14.4 \mathrm{~Hz}, 2 \mathrm{H}), 3.77-3.62(\mathrm{~m}, 4 \mathrm{H})$. ${ }^{13} \mathrm{C}$ NMR $\left(75 \mathrm{MHz}, \mathrm{CDCl}_{3}\right.$, ppm) $\delta 153.6,137.8,128.0,127.0,126.7,121.0,109.8,44.4$, 37.2. FT-IR (thin film, $\mathrm{cm}^{-1}$ ) 3060, 2916, 1703, 1611, 1492, 1422, 1393, 1291, 1159, 1034, 890. High resolution MS (EI, $m / z$ ): anal calcd for $\mathrm{C}_{17} \mathrm{H}_{16} \mathrm{~N}_{2} \mathrm{~S}_{2} \mathrm{O}$ : 328.0699; found: 328.0699; error: 0 ppm.

\section{3,9-Dithia-benzimidazolophane 8B}

Into an argon-flushed $50 \mathrm{~mL}$ rb flask equipped with magnetic stirbar and rubber septum was placed $44 \mathrm{mg}(0.2 \mathrm{mmol})$ 1,3-bis(methoxymethyl)-2-benzimidazolidinone, $34 \mathrm{mg}(0.2 \mathrm{mmol}) 1,3$ - bismercaptomethyl - 4, 6-dimethylbenzene ${ }^{5}$ in $10 \mathrm{~mL} \mathrm{CH}_{2} \mathrm{Cl}_{2}$ at room temperature to which $0.1 \mathrm{~mL} \mathrm{BF}_{3} \mathrm{OEt}_{2}(0.113 \mathrm{~g}, 0.8 \mathrm{mmol})$ was added dropwise via syringe. The reactants were stirred for $4 \mathrm{~h}$ at room temperature at which time the reaction was complete (TLC). The reaction contents were poured into a separatory funnel containing $50 \mathrm{~mL} \mathrm{NaHCO}$, partitioned, and the aqueous layer was extracted three times with $20 \mathrm{~mL}$ portions of $\mathrm{CH}_{2} \mathrm{Cl}_{2}$. The pooled organics were dried $\left(\mathrm{K}_{2} \mathrm{CO}_{3}\right)$ and concentrated to give a white foam which was purified by flash chromatography $(1 \mathrm{~cm} \mathrm{x}$ $12 \mathrm{~cm}, 1: 4$ ethyl acetate-hexanes $(100 \mathrm{~mL})$ then gradient elution to 1:1 ethyl acetatehexanes) to afford $20 \mathrm{mg} \mathbf{8 B}$ as a white solid (29\%). Crystallization from $\mathrm{CH}_{2} \mathrm{Cl}_{2}$ gave white crystals, mp $206-208{ }^{\circ} \mathrm{C}$. Analytical TLC: (1:3 ethyl acetate-hexanes) $\mathrm{R}_{f} 0.45 .{ }^{1} \mathrm{H}$ NMR (500 MHz, $\left.\mathrm{CDCl}_{3}, \mathrm{ppm}\right) \delta 7.00-6.97(\mathrm{~m}, 3 \mathrm{H}), 6.90-6.88(\mathrm{~m}, 2 \mathrm{H}), 6.33(\mathrm{~s}, 1 \mathrm{H})$, $5.91(\mathrm{~d}, J=14.5 \mathrm{~Hz}, 2 \mathrm{H}), 4.49(\mathrm{~d}, J=14.5 \mathrm{~Hz}, 2 \mathrm{H}), 3.80(\mathrm{~d}, J=15 \mathrm{~Hz}, 2 \mathrm{H}), 3.72(\mathrm{~d}, J=$ $15 \mathrm{~Hz}, 2 \mathrm{H}), 2.06(\mathrm{~s}, 6 \mathrm{H}) .{ }^{13} \mathrm{C} \mathrm{NMR}\left(75 \mathrm{MHz}, \mathrm{CDCl}_{3}, \mathrm{ppm}\right) \delta 153.7,134.7,132.6(\mathrm{CH})$, 131.6, 128.0 (CH), 127.1, $120.8(\mathrm{CH}), 109.9,44.3\left(\mathrm{CH}_{2}\right), 36.0\left(\mathrm{CH}_{2}\right), 18.3\left(\mathrm{CH}_{3}\right)$. FT-IR (thin film, $\mathrm{cm}^{-1}$ ) 1701, 1559, 1492, 1425, 1394, 1286, 1034. High resolution MS (EI,

${ }^{5}$ Gerisch, M.; Krumper, J. R.; Bergman, R. G.; Tilley, T. D. Organometallics 2003, 22, 47-58. 
m/z): anal calcd for $\mathrm{C}_{19} \mathrm{H}_{20} \mathrm{~N}_{2} \mathrm{~S}_{2} \mathrm{O}$ : 356.10126; found: 356.1012; error: 0.3 ppm.

\section{[2.2] Benzimidazolophane 9A}

Into an immersion well reactor was dissolved $1.3 \mathrm{~g}$ crude $\mathbf{8 A}$ in $200 \mathrm{~mL}$ of $2: 3 \mathrm{v} / \mathrm{v}$ benzene- $\mathrm{P}(\mathrm{OEt})_{3}$ which was equipped with a medium pressure $\mathrm{Hg}$ lamp (Hanovia) contained in a pyrex water-jacketed cold finger. The solution was continuously sparged with a stream of argon and was irradiated for $5 \mathrm{~h}$. The solvents were removed in vacuo (high vacuum) and the crude was purified by flash chromatography $(2.5 \mathrm{~cm} \times 12 \mathrm{~cm}, 1: 4$ ethyl acetate-hexanes) affording $250 \mathrm{mg}(25 \%)$ of $\mathbf{9 A}$. Crystals of 9A were obtained by slow evaporation of $\mathrm{CH}_{2} \mathrm{Cl}_{2}, \mathrm{mp} 148-149^{\circ} \mathrm{C}$. ${ }^{1} \mathrm{H}$ NMR $\left(300 \mathrm{MHz}, \mathrm{CDCl}_{3}, \mathrm{ppm}\right) \delta 7.33$ $-7.10(\mathrm{~m}, 7 \mathrm{H}), 5.20(\mathrm{~s}, 1 \mathrm{H}), 4.04-3.85(\mathrm{~m}, 4 \mathrm{H}), 2.85-2.66(\mathrm{~m}, 4 \mathrm{H}) .{ }^{13} \mathrm{C}$ NMR $(75.5$ $\left.\mathrm{MHz}, \mathrm{CDCl}_{3}, \mathrm{ppm}\right) \delta 158.2,138.5,131.7,131.4,129.9,126.6,121.8,109.9,45.7,33.8$. FT-IR (thin film, $\mathrm{cm}^{-1}$ ) 2942, 2360, 1718, 1486, 1439, 1372, 1345, 1298, 1142, 1013, 786, 749, 719. High resolution MS (E/I, $m / z)$ : anal calcd for $\mathrm{C}_{17} \mathrm{H}_{16} \mathrm{~N}_{2} \mathrm{O}: 264.1257$; found: 264.1261; error: 1.6 ppm.

Crystals of 9A were subjected to x-ray analysis. See attached cif file.

\section{[2.2] Benzimidazolophane 9B}

Into an immersion well reactor was dissolved $1.5 \mathrm{~g} \mathbf{8 B}$ in $250 \mathrm{~mL}$ of $2: 3 \mathrm{v} / \mathrm{v}$ benzene- $\mathrm{P}(\mathrm{OEt})_{3}$ which was equipped with a medium pressure $\mathrm{Hg}$ lamp (Hanovia) contained in a pyrex water-jacketed cold finger. The solution was continuously sparged with a stream of argon and was irradiated for $3 \mathrm{~h}$. The volatiles were removed in vacuo (high vacuum) and the crude product was purified by flash chromatography $(2.5 \mathrm{~cm} \times 12$ $\mathrm{cm}, 1: 4$ ethyl acetate-hexanes) affording $134 \mathrm{mg}(11 \%)$ of 9B. Crystals of 9B were obtained by slow evaporation of $\mathrm{CH}_{2} \mathrm{Cl}_{2}$, mp $177-179{ }^{\circ} \mathrm{C}$. ${ }^{1} \mathrm{H}$ NMR $\left(500 \mathrm{MHz}, \mathrm{CDCl}_{3}\right.$, ppm) $\delta 7.17-7.14(\mathrm{~m}, 2 \mathrm{H}), 7.11-7.09(\mathrm{~m}, 2 \mathrm{H}), 6.96(\mathrm{~s}, 1 \mathrm{H}), 5.00(\mathrm{~s}, 1 \mathrm{H}), 3.96-3.93$ $(\mathrm{m}, 2 \mathrm{H}), 3.87-3.81(\mathrm{~m}, 2 \mathrm{H}), 2.86(\mathrm{~d}, J=13.5 \mathrm{~Hz}, 2 \mathrm{H}), 2.58-2.52(\mathrm{~m}, 2 \mathrm{H}), 2.306(\mathrm{~s}$, $6 \mathrm{H}) .{ }^{13} \mathrm{C}$ NMR $\left(75.5 \mathrm{MHz}, \mathrm{CDCl}_{3}, \mathrm{ppm}\right) \delta 158.3,134.2,134.0,132.4(\mathrm{CH}), 132.2(\mathrm{CH})$, 131.5, $121.6(\mathrm{CH}), 109.9(\mathrm{CH}), 44.4\left(\mathrm{CH}_{2}\right), 30.2\left(\mathrm{CH}_{2}\right), 18.4\left(\mathrm{CH}_{3}\right)$. FT-IR (thin film, $\mathrm{cm}^{-}$ $\left.{ }^{1}\right)$ 2940, 2868, 1719, 1487, 1446, 1374, 1341, 1152, 1013, 748. High resolution MS (E/I, $\mathrm{m} / \mathrm{z}$ ): anal calcd for $\mathrm{C}_{19} \mathrm{H}_{20} \mathrm{~N}_{2} \mathrm{O}$ : 292.1570; found: 292.1575; error: $1.7 \mathrm{ppm}$. 


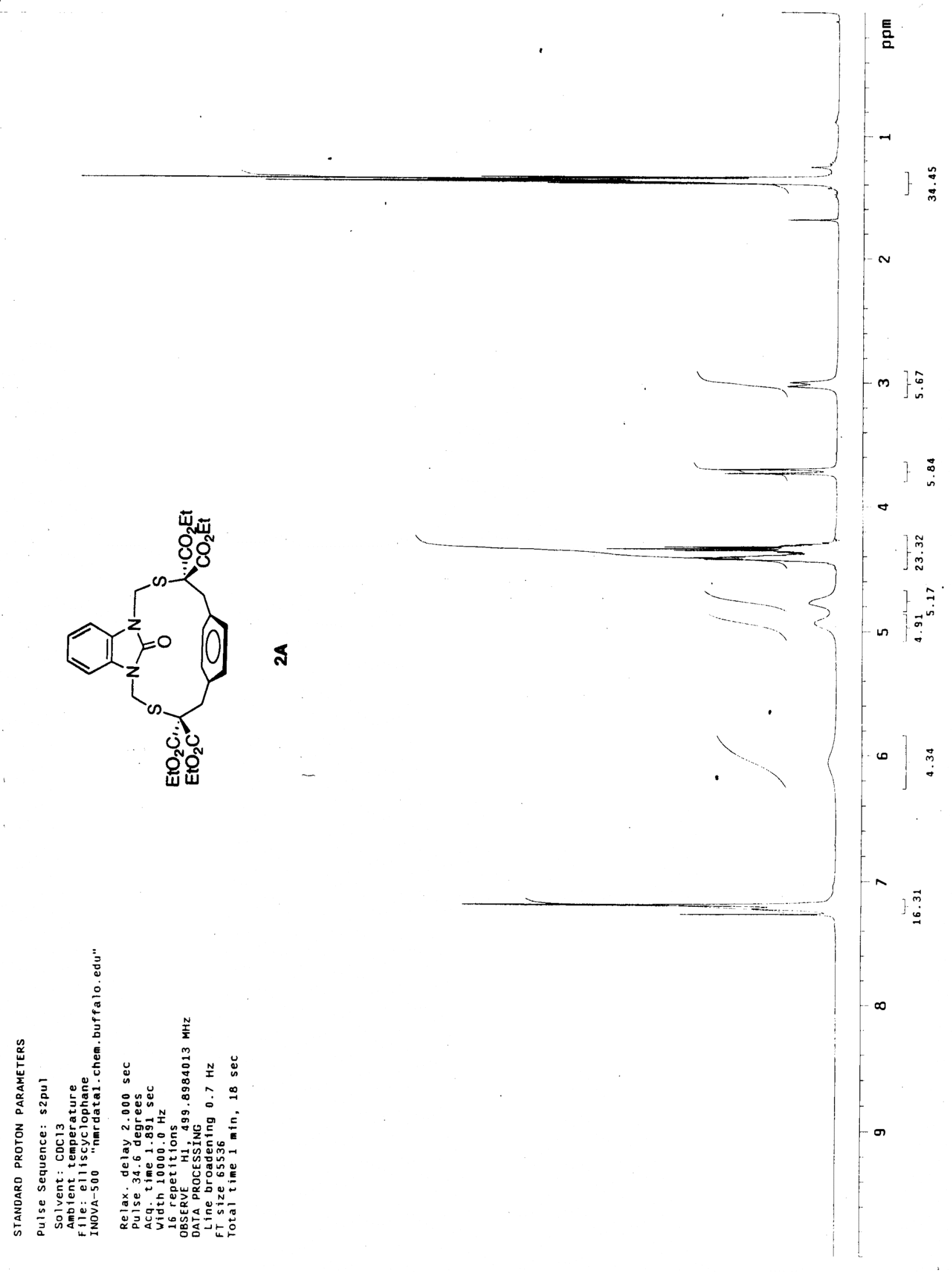



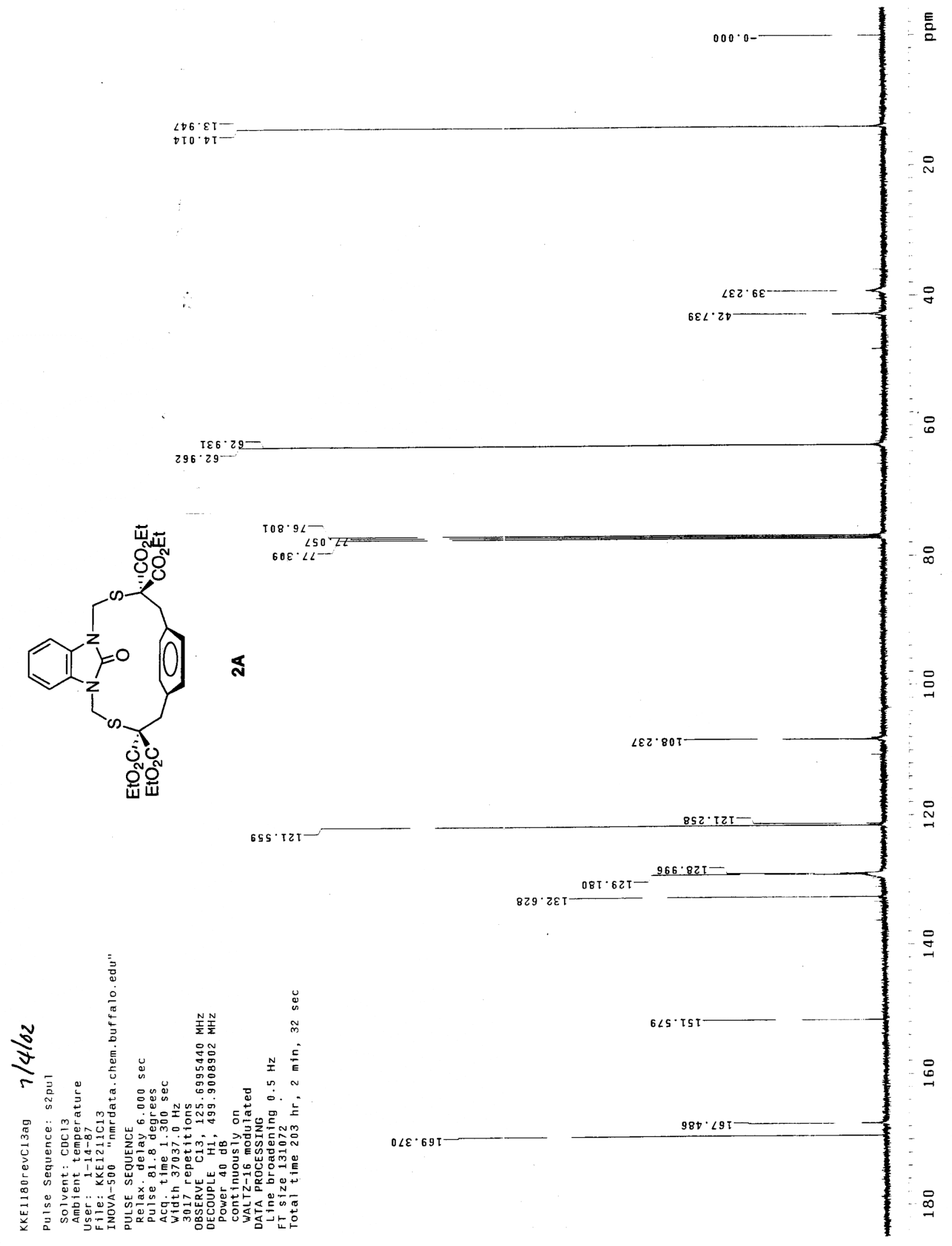


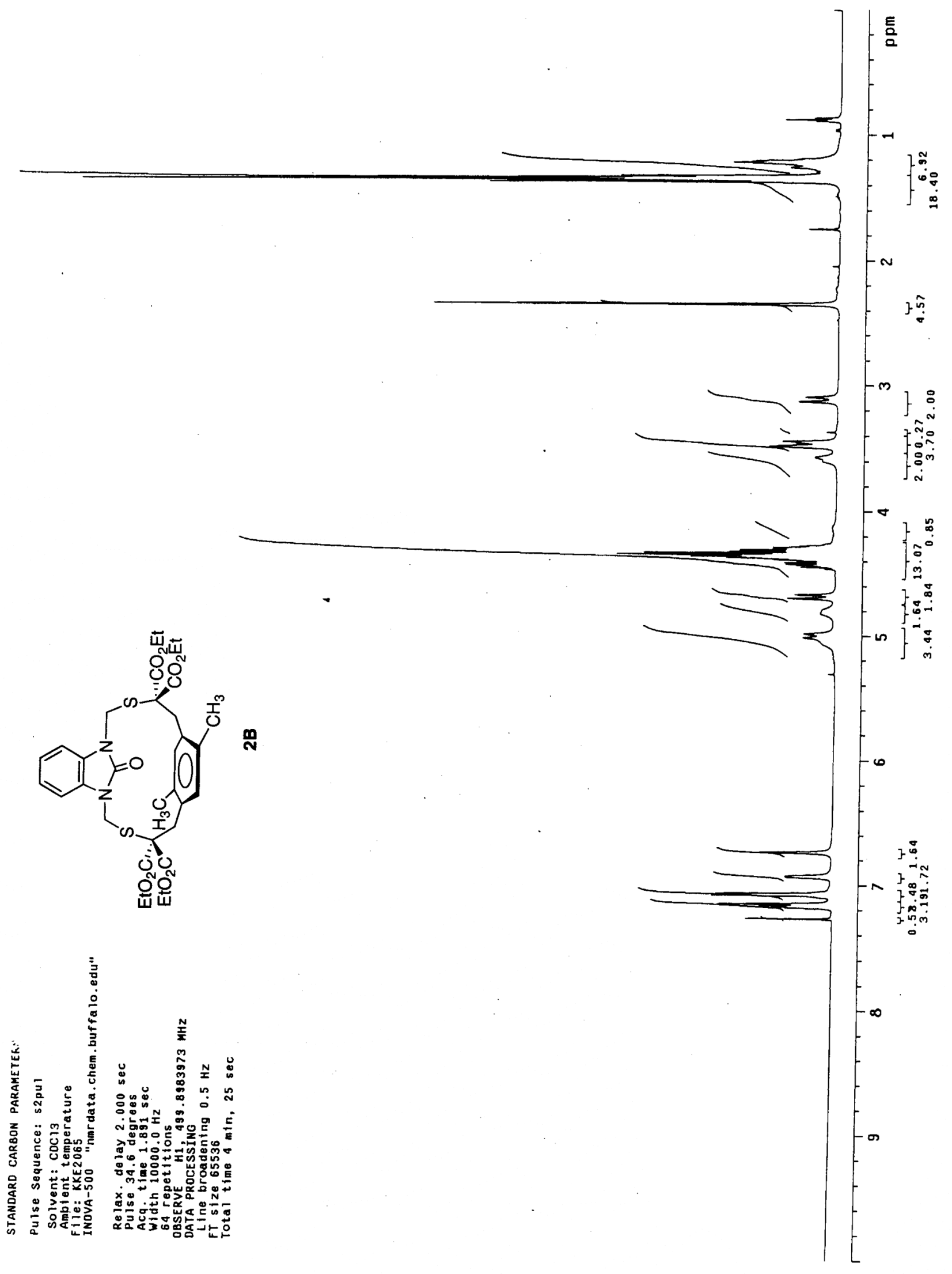




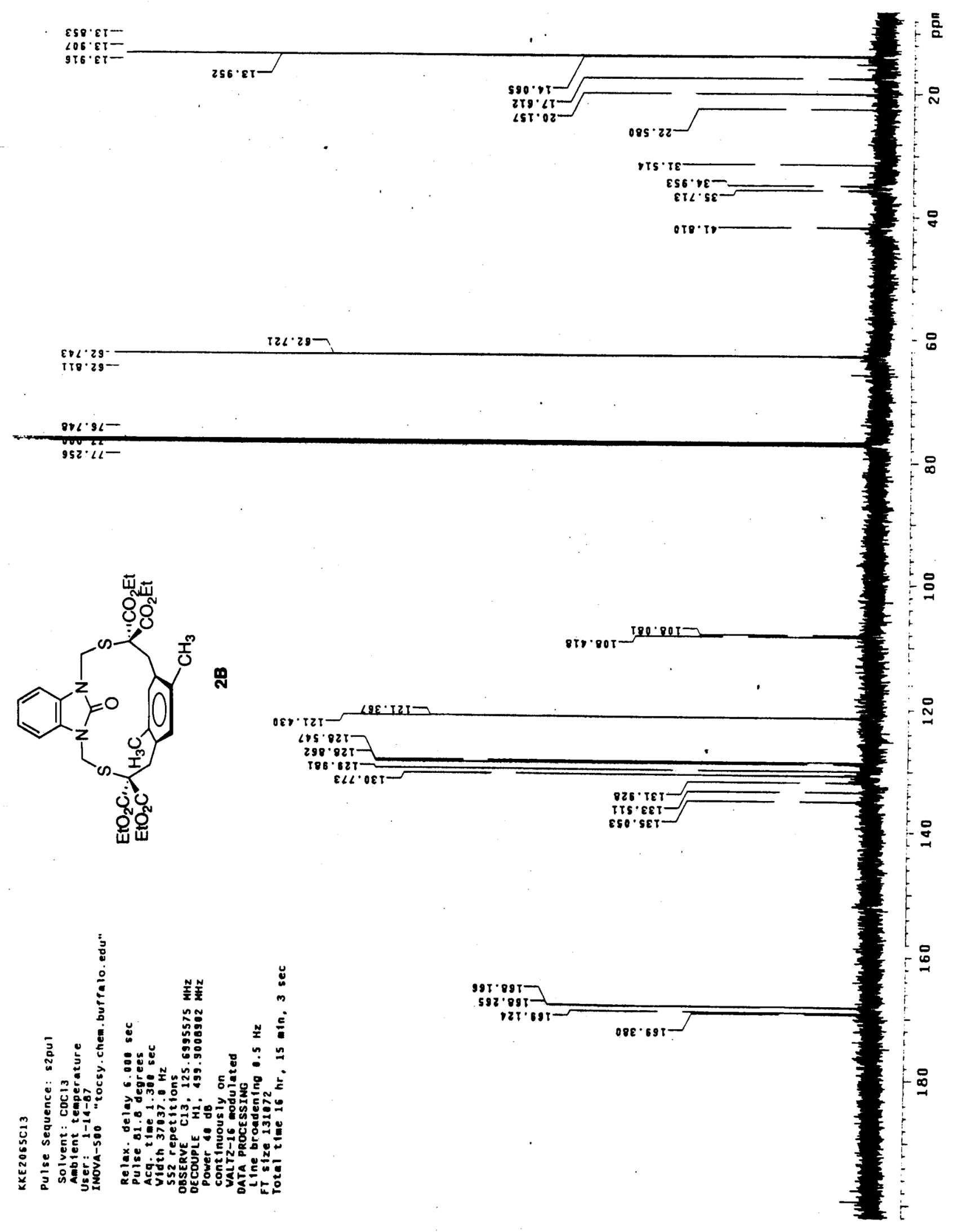




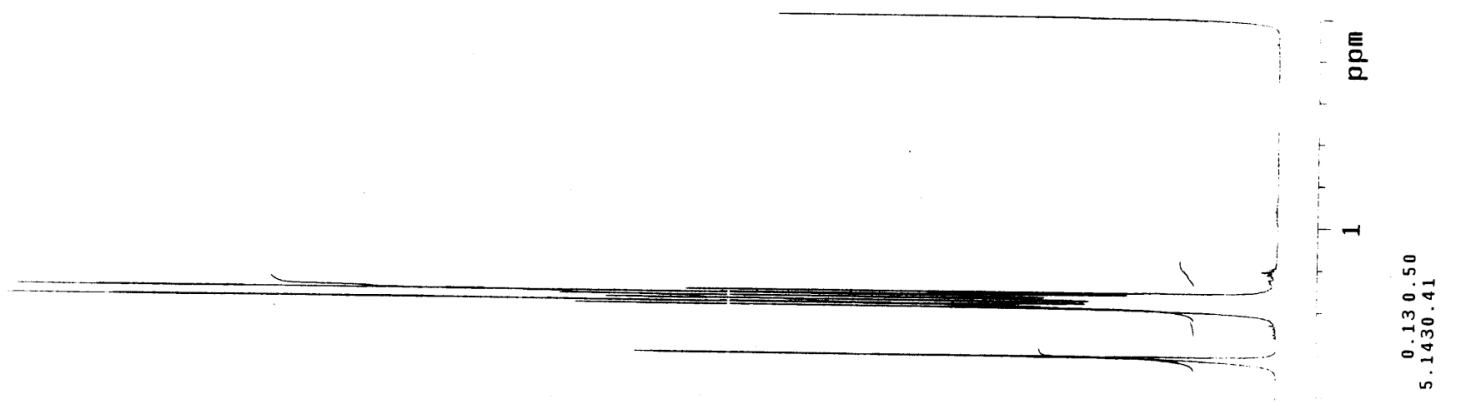

$N$
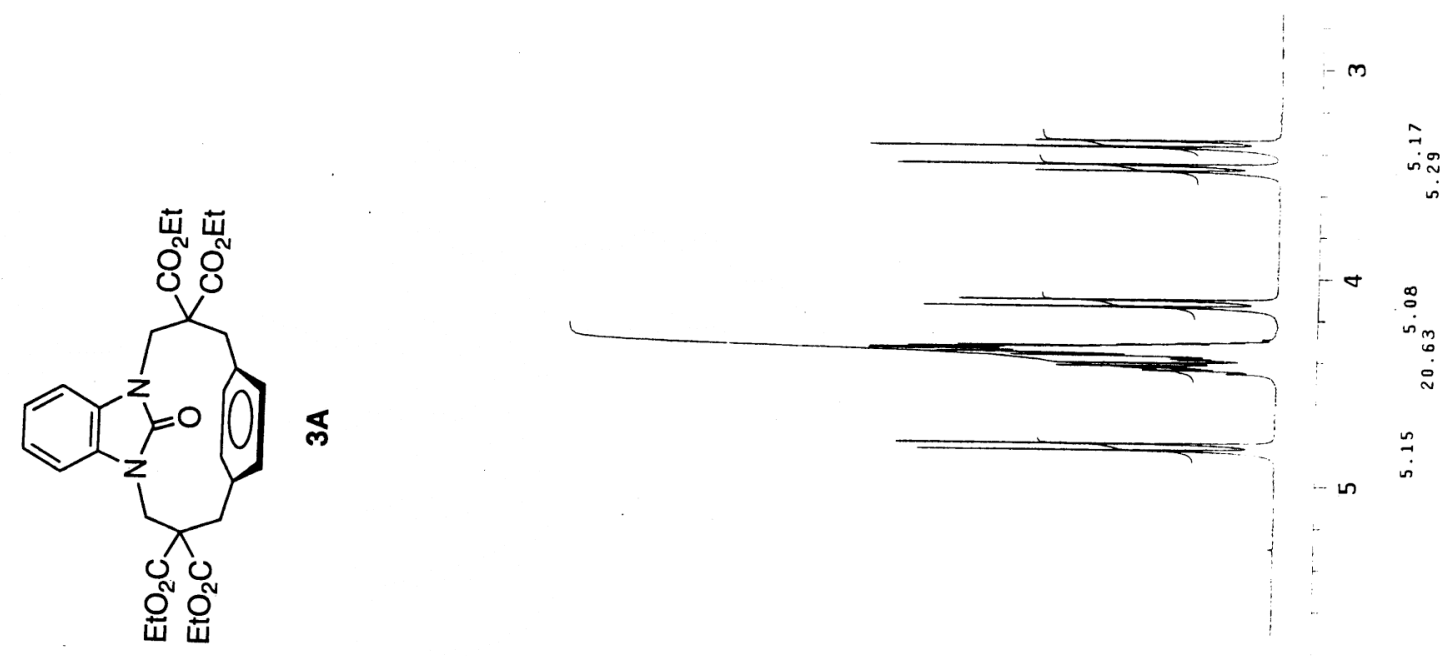

๑
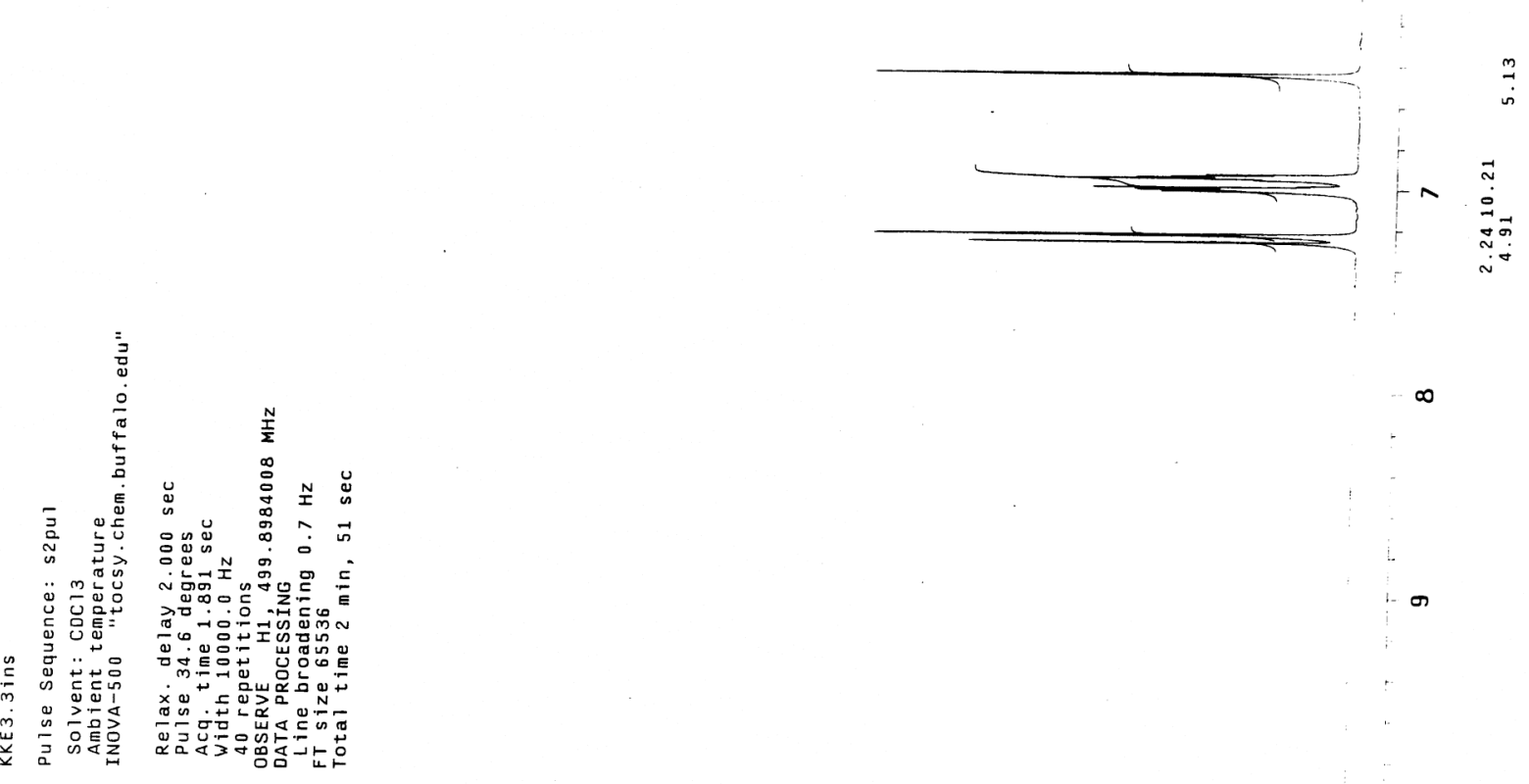

$\infty$

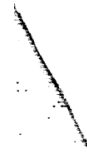



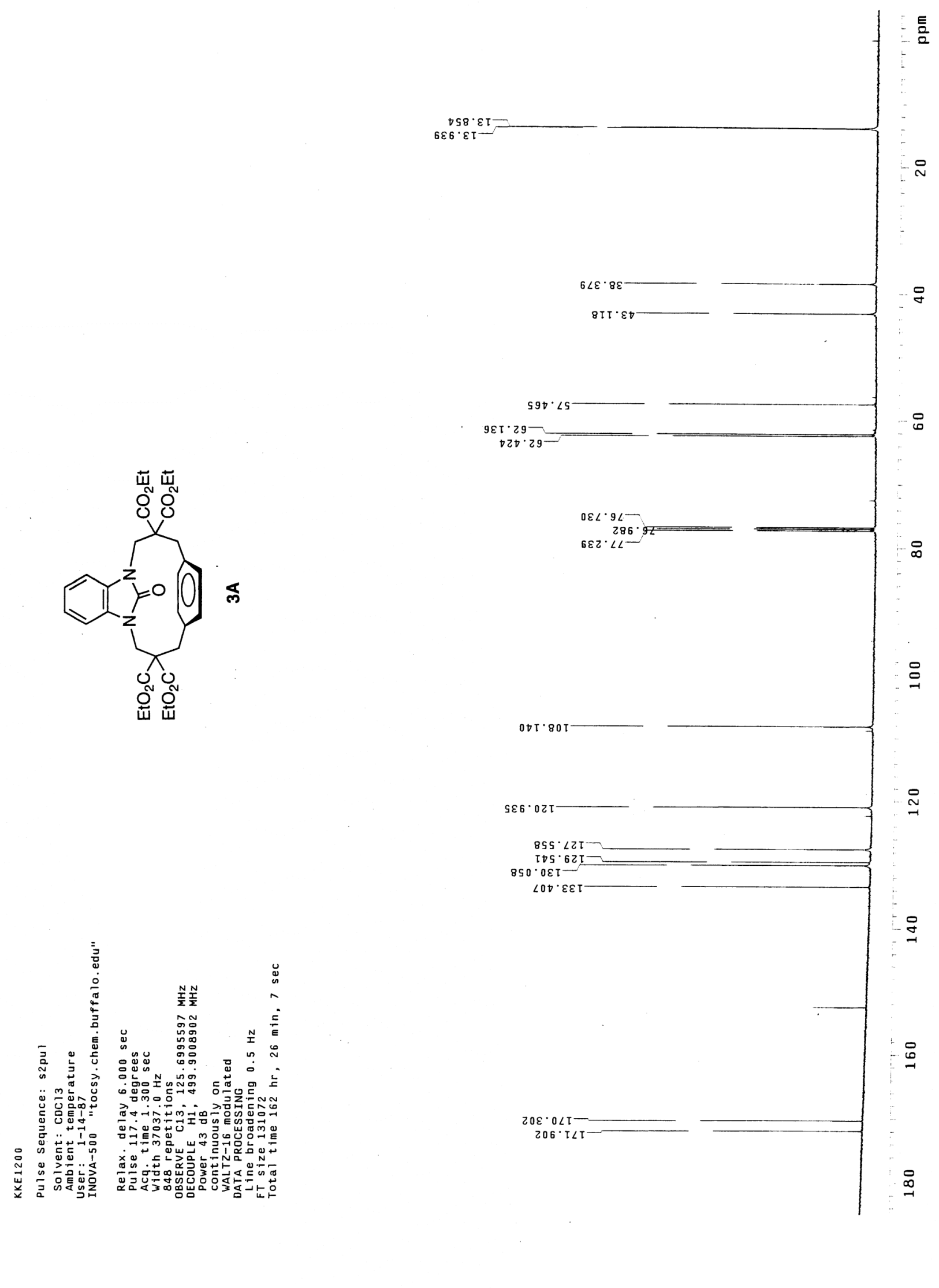


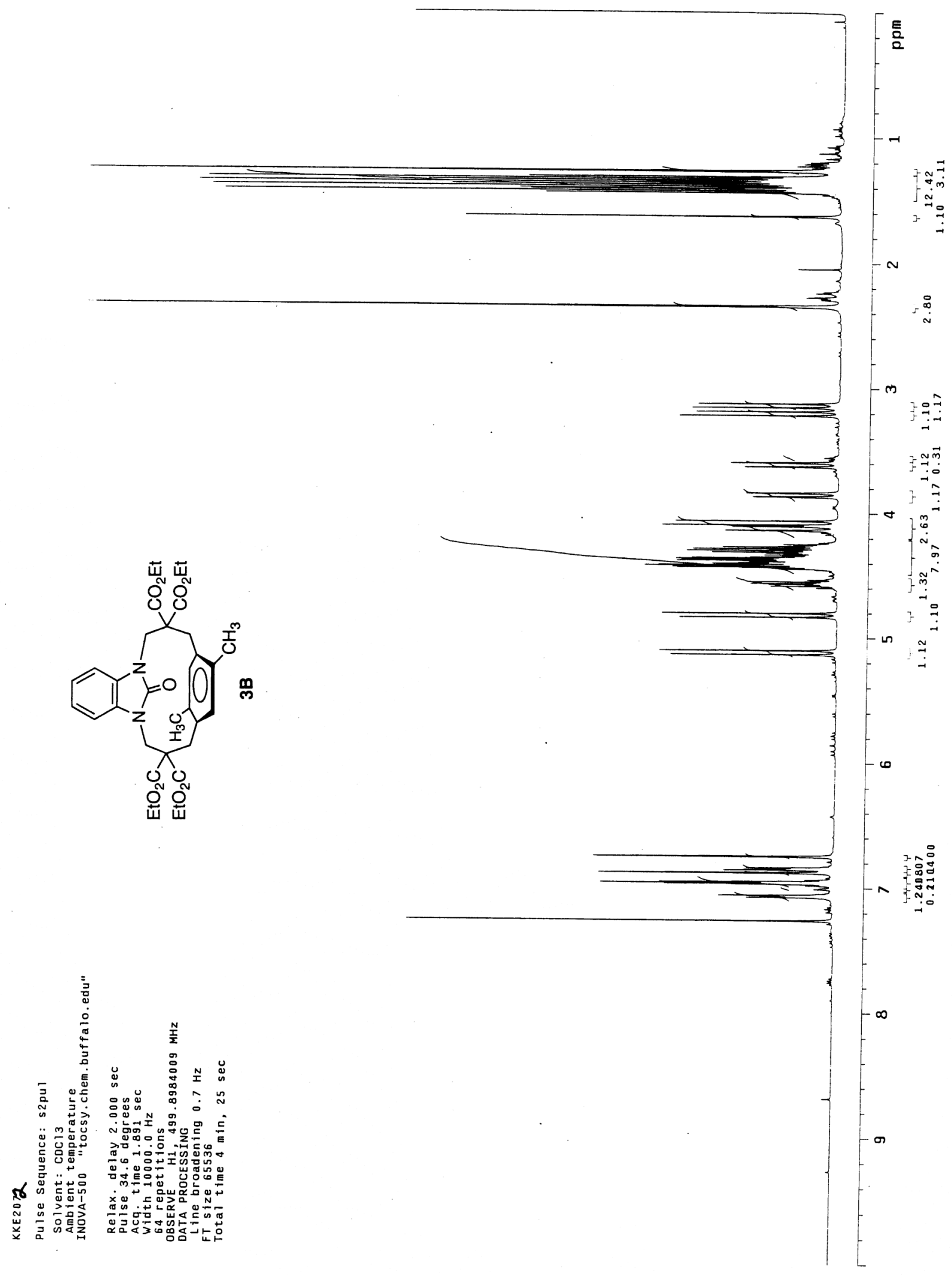




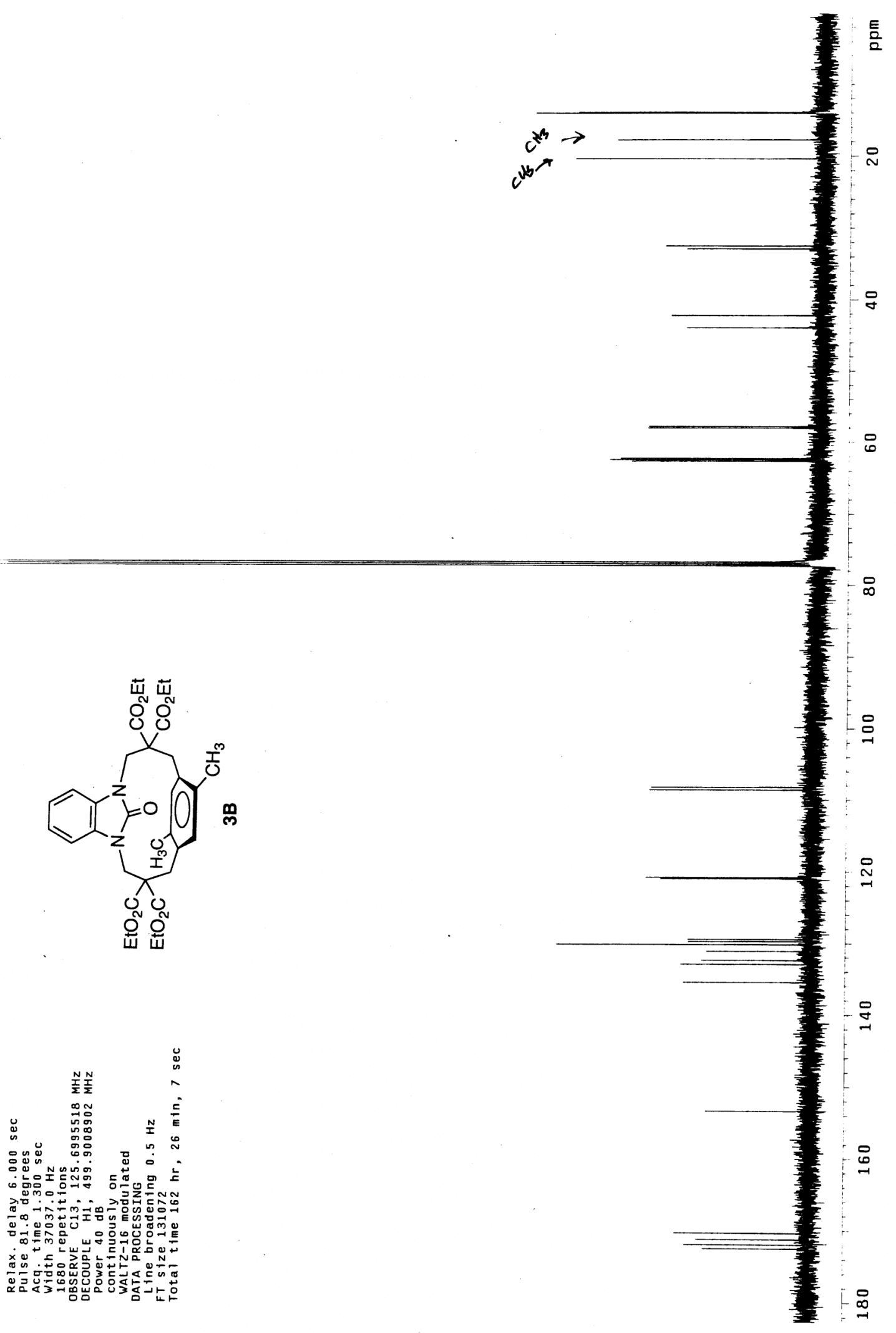




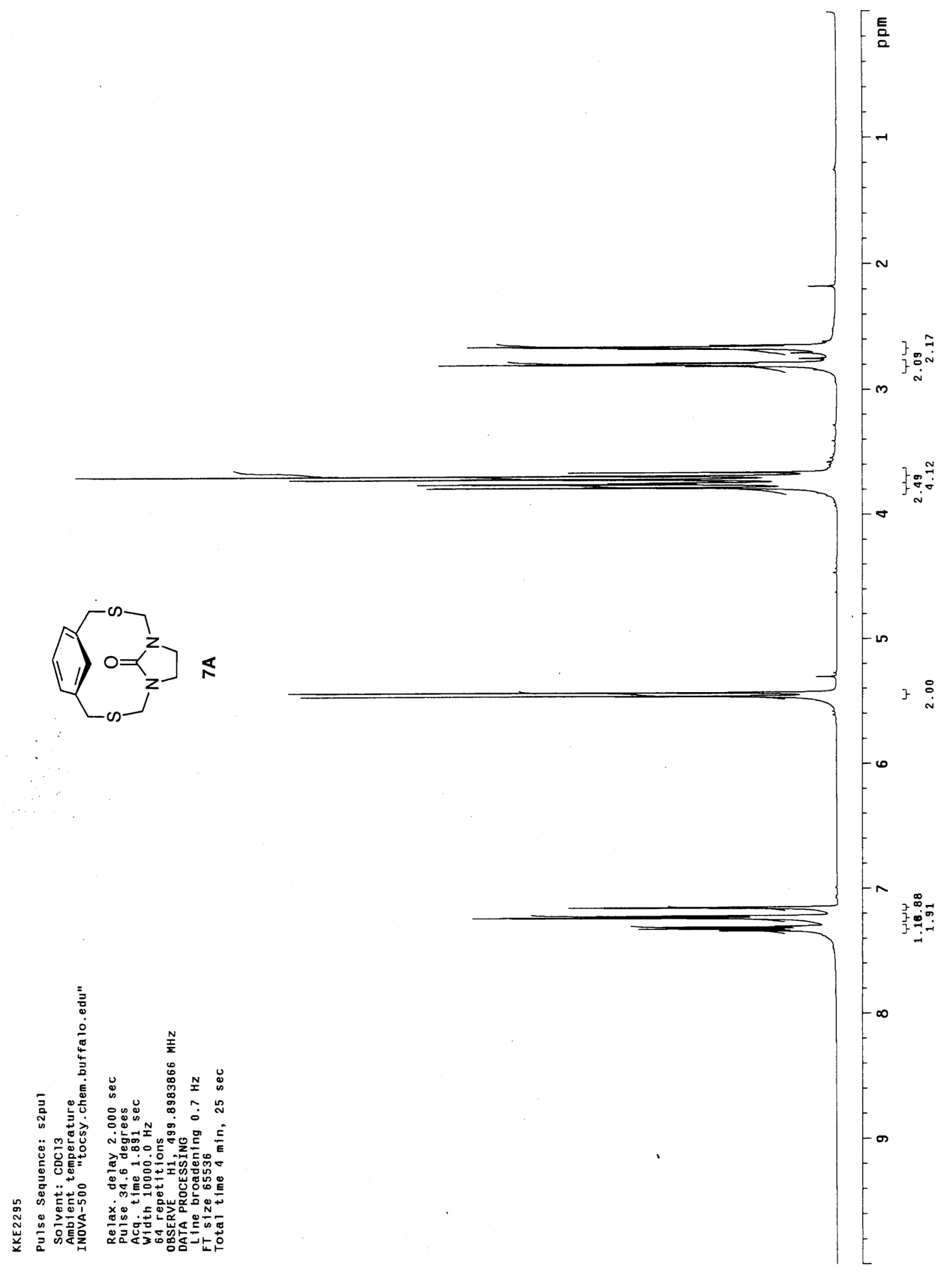




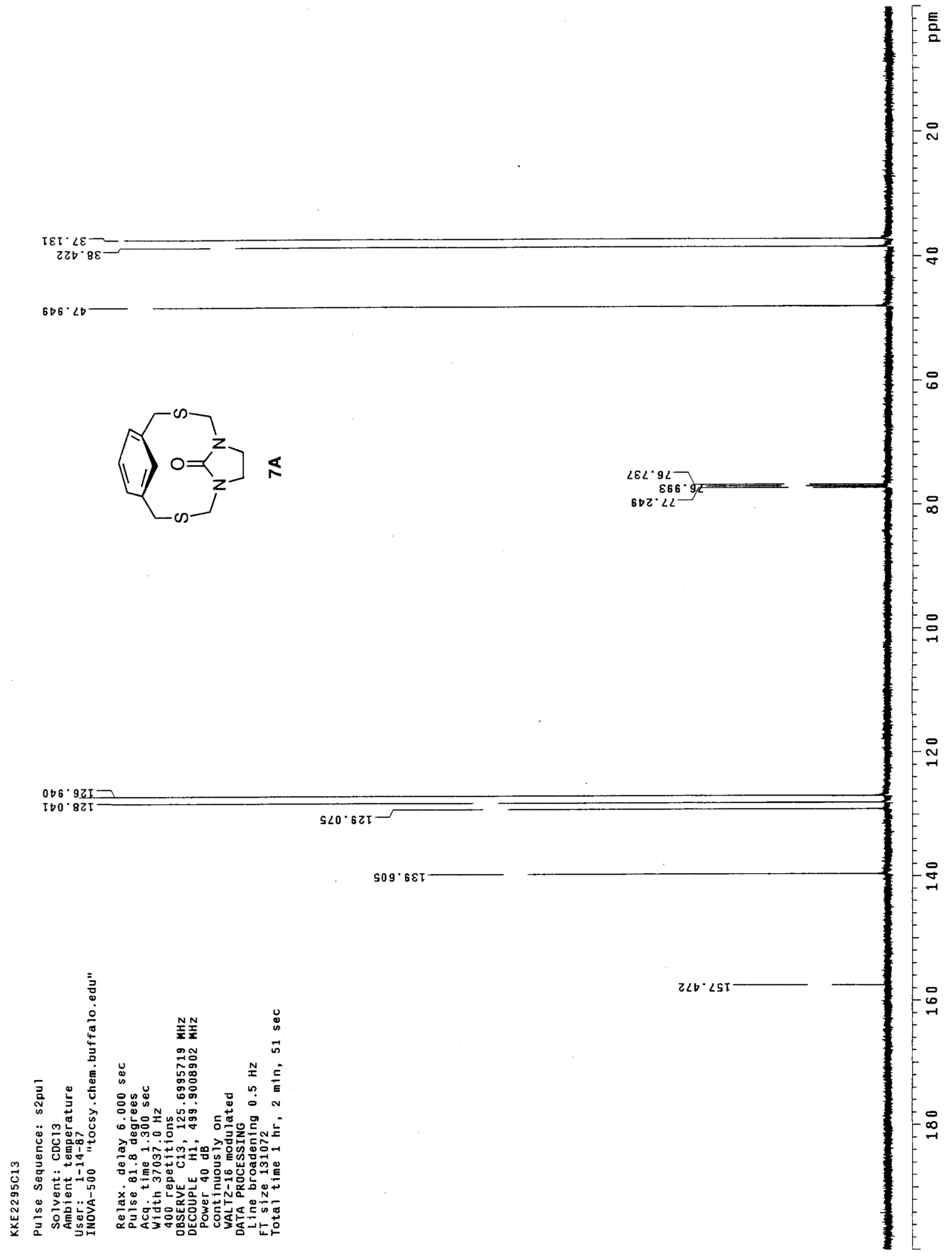




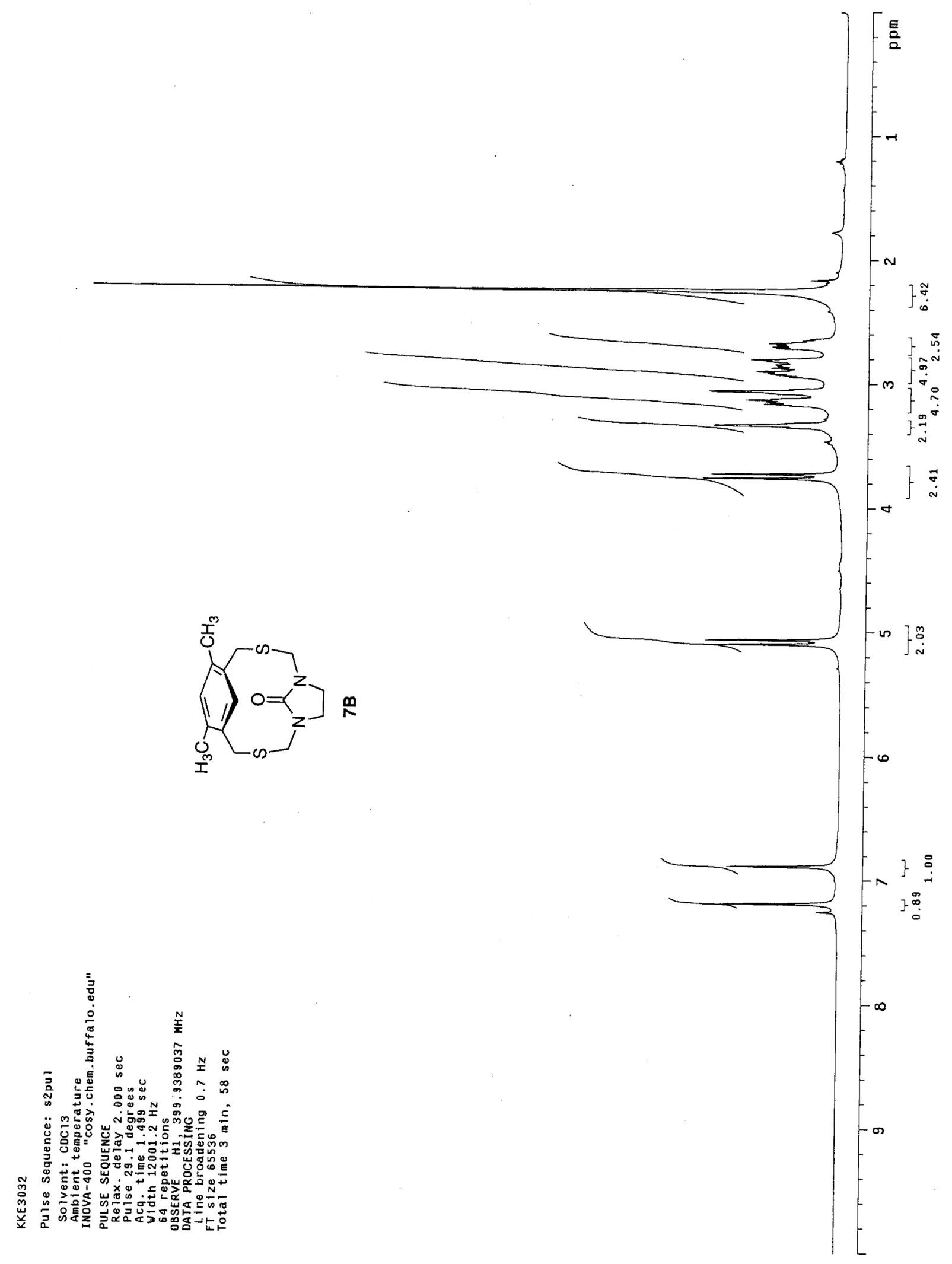




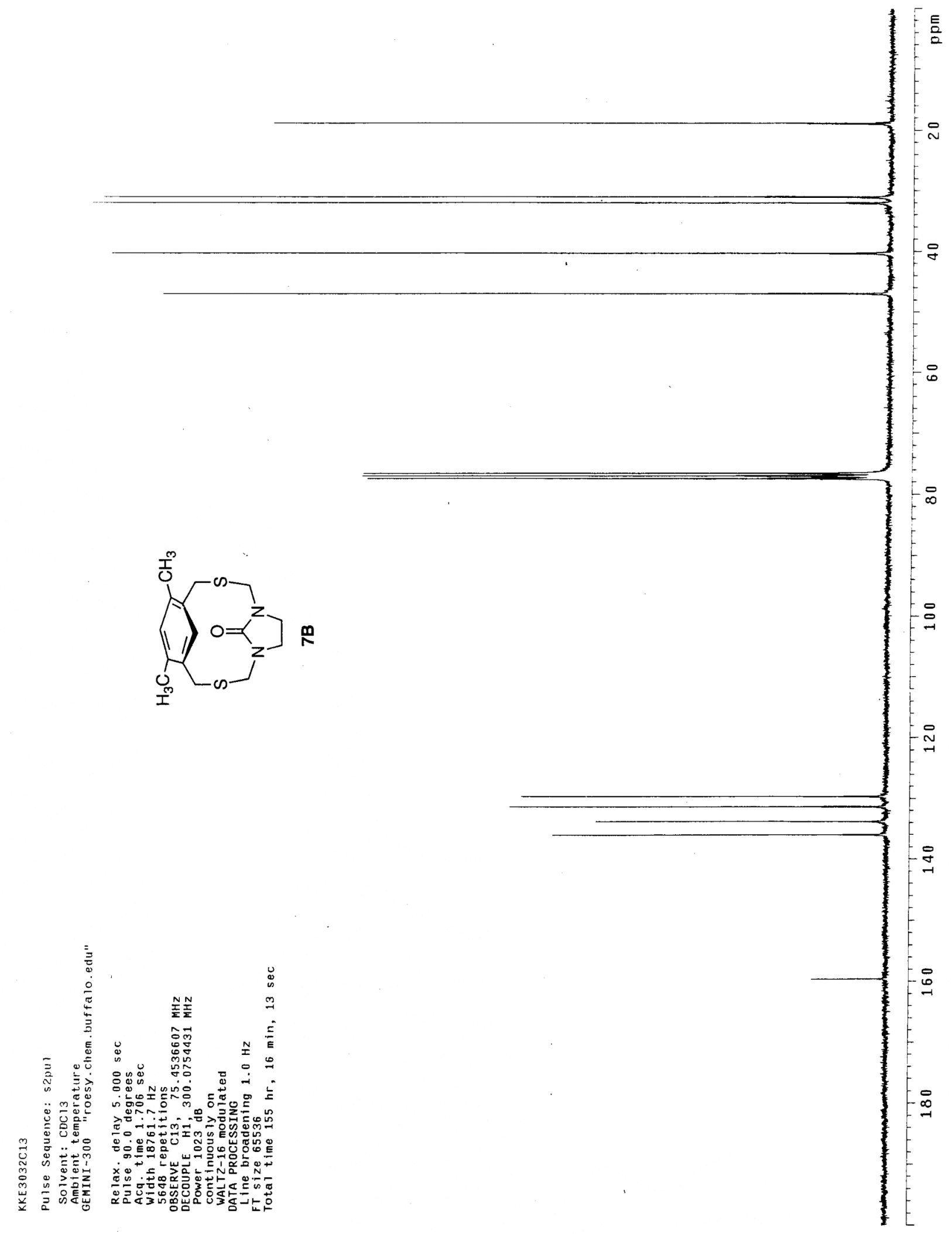



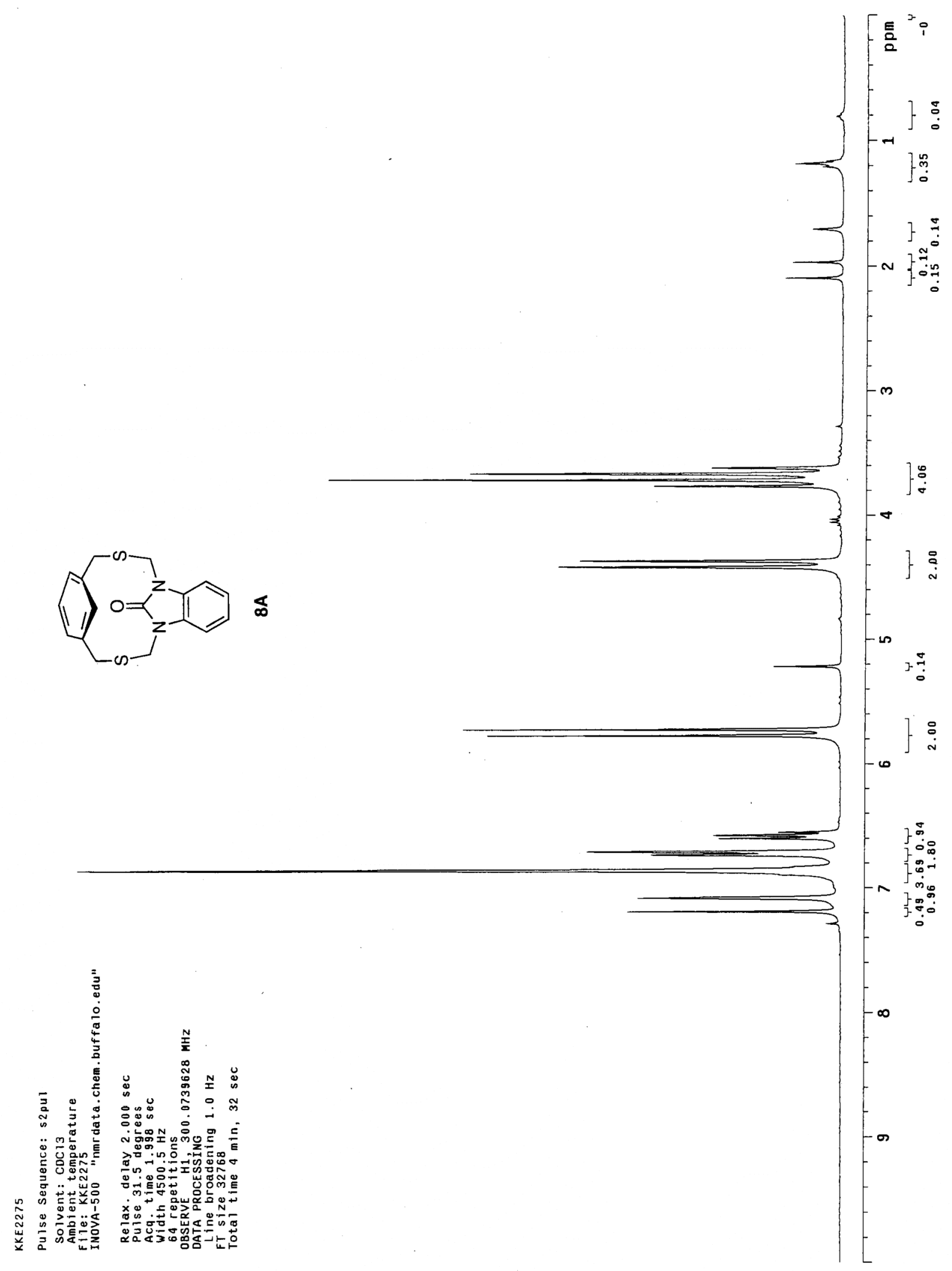


$$
\text { if }
$$



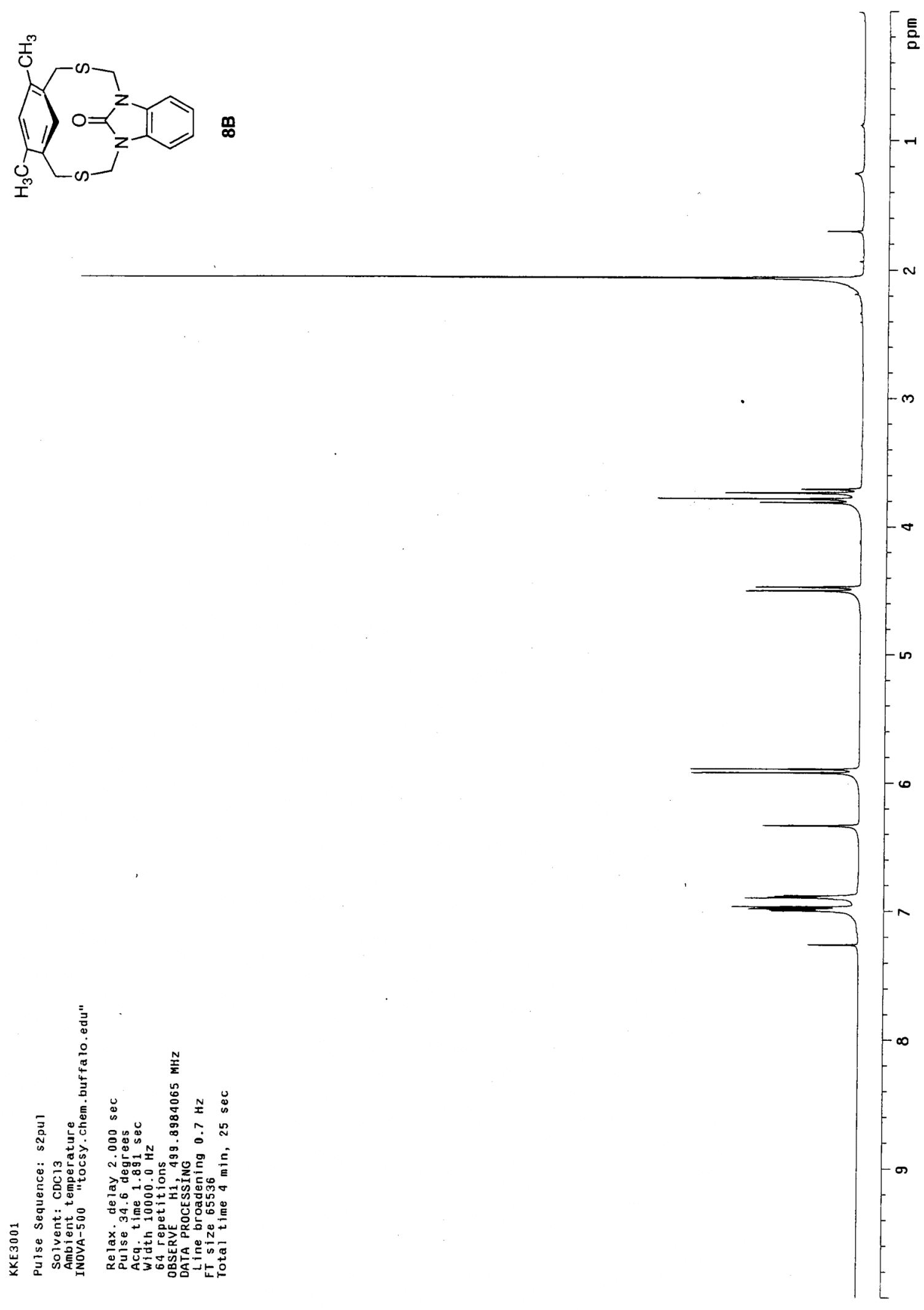


$$
\text { it }
$$




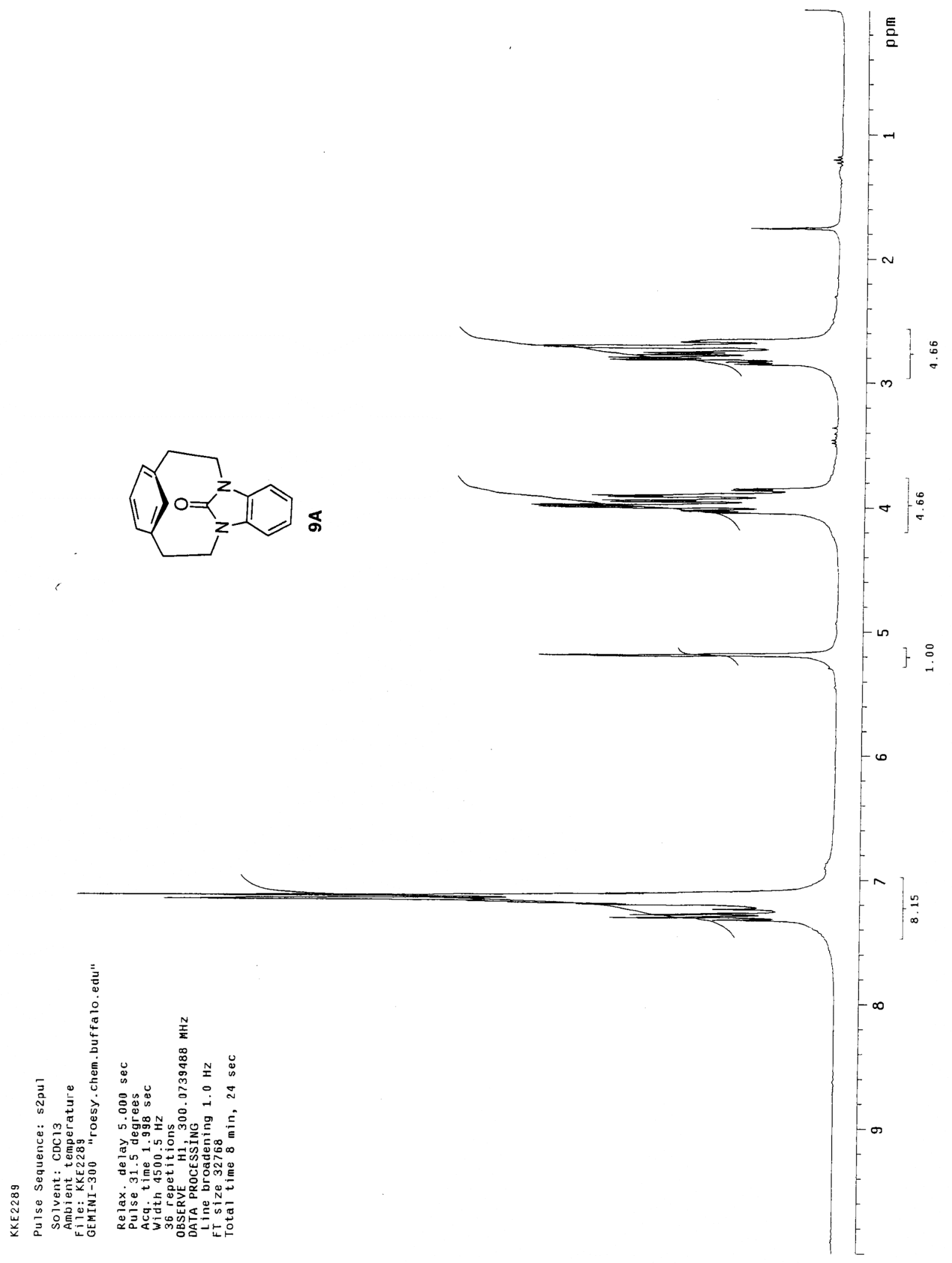




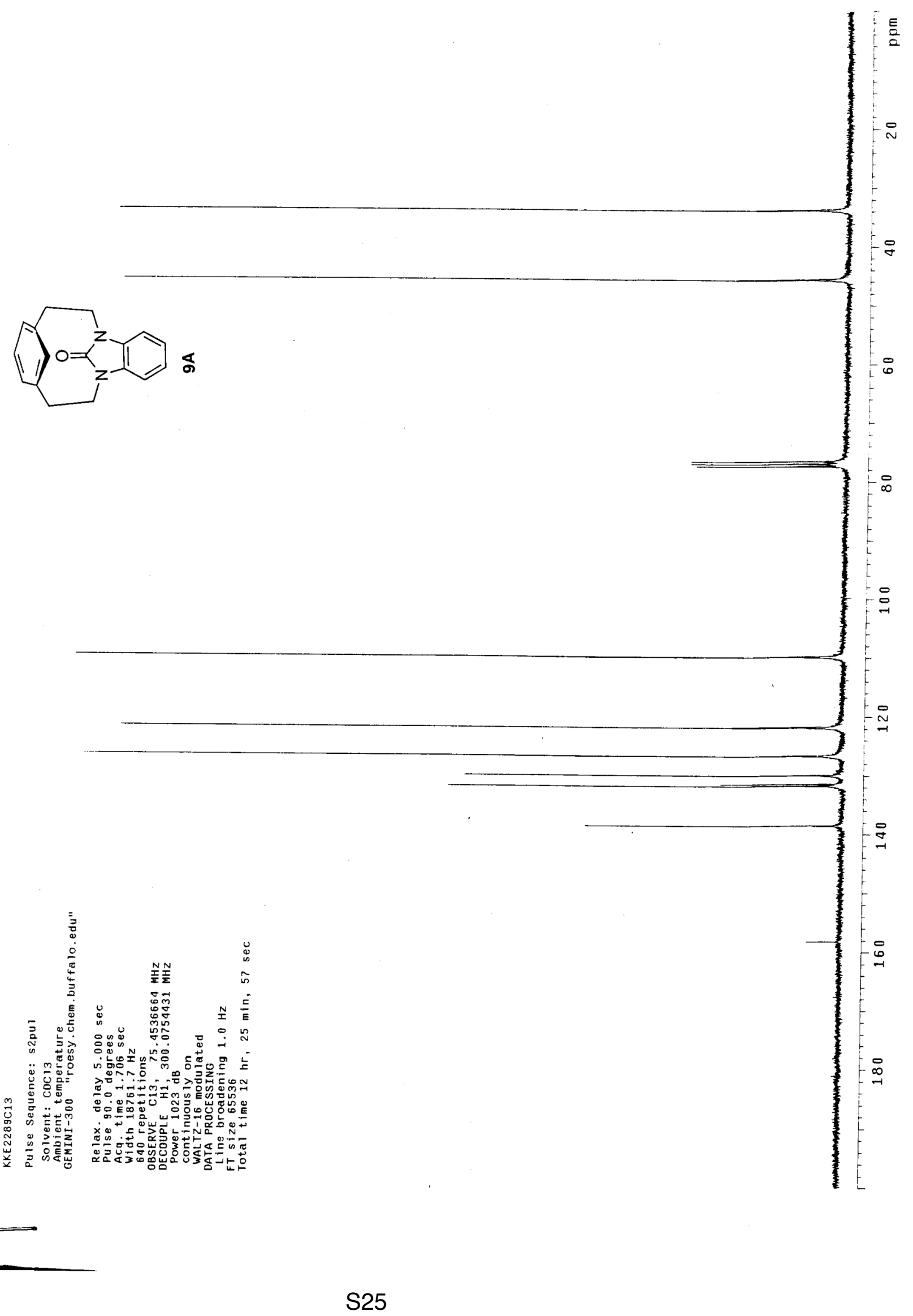




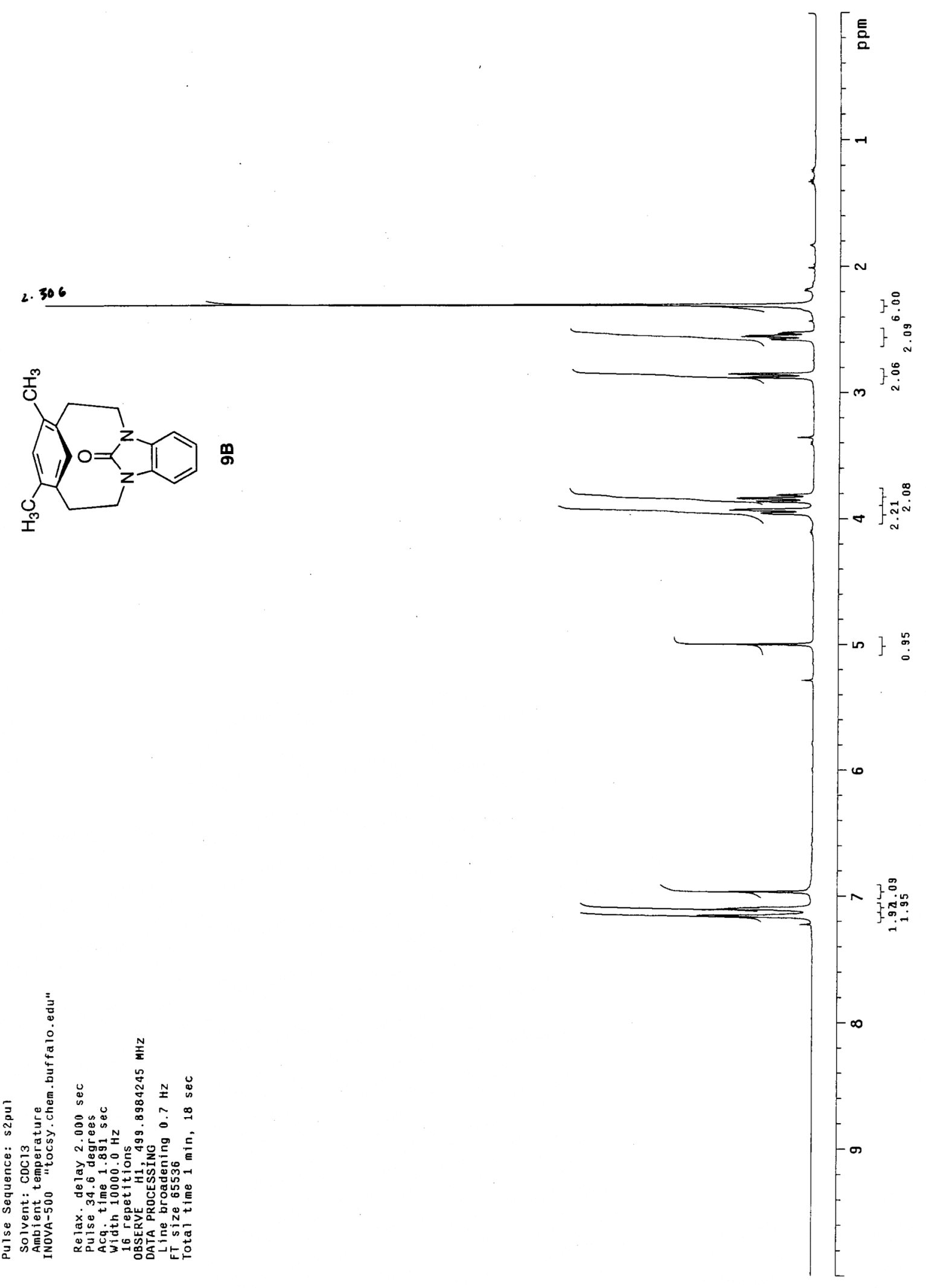




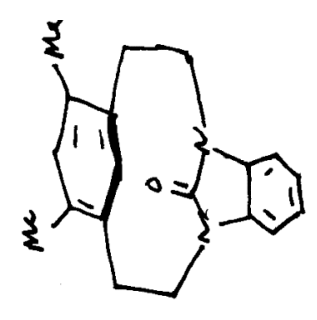

$\varepsilon \tau \tau^{\circ} 0 \varepsilon^{-}$
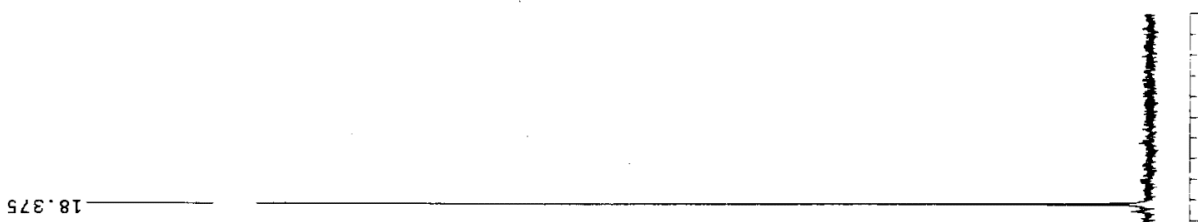

$\angle 20 \cdot 0 b-$

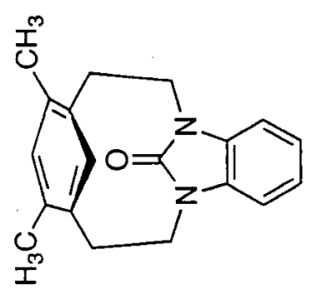

용
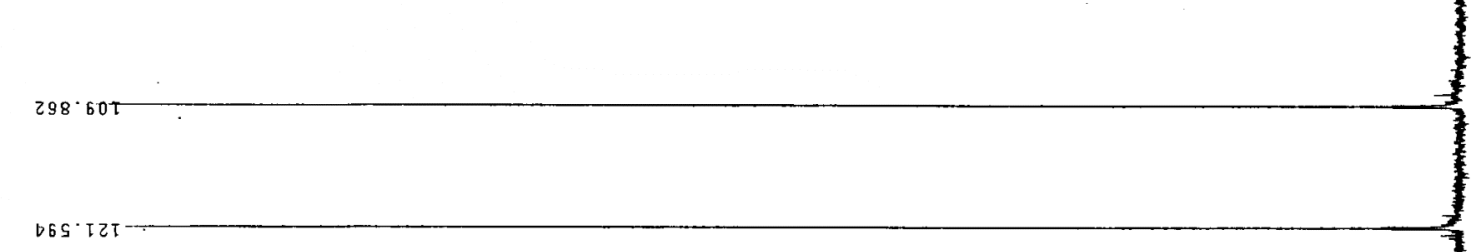

$\cong$

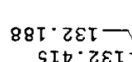

OZS. TEI -

SID $Z E I$

$2 \varepsilon 0^{\circ} \circ \varepsilon$

$90 \tau^{\circ} \downarrow \varepsilon \tau$
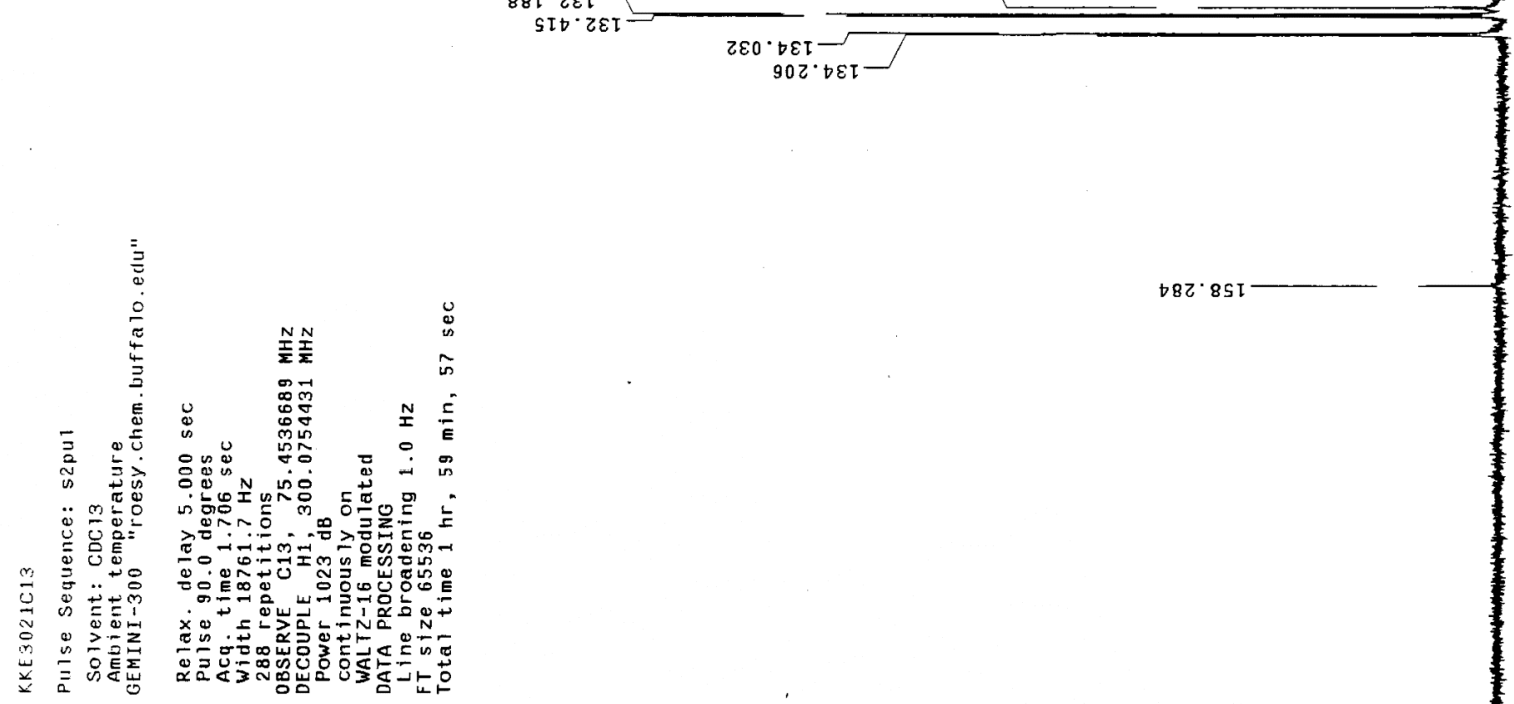

$\frac{1}{8}$

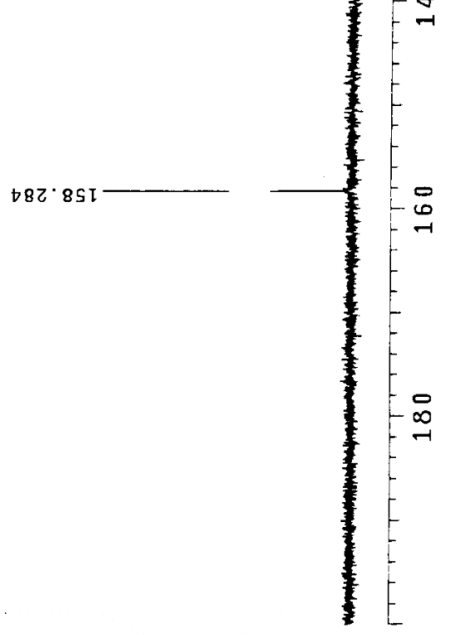

